\title{
Die Nesselkapseln der Aktinien und ihre differentialdiagnostische Bedeutung
}

\author{
H. SCHMIDT \\ I. Zoologisches Institut der Universität Gießen, Gießen/Lahn
}

\begin{abstract}
ABST'RACT: The nematocysts of the sea anemones and their importance for differentialdiagnosis. Nematocysts of 36 actinian species of the North Sea, Mediterranean and Red Sea were studied by phase contrast and electron microscopy. Spirocysts differ from nematocysts in having, just after discharge, a single thread of secretion ("Quellfaden") entwining the tube in left turns. Haplonemes and heteronemes are redefined. In undischarged condition, heteronemes have a basal shaft ("Achsenkörper") of greater diameter than the thread. The everted shaft of heteronemes bears coarse armature abruptly reduced on the thread. The tube of haplonemes, however, is of nearly uniform diameter (isorhizes) or broader at the basis (anisorhizes). The length of the spines is proportional to the respective diameter of the tube. Other categories of haplonemes are eliminated. Haplonemes have a wider distribution than generally believed. They are not known as yet from the scapus of many Acontiaria and from the tentacles of some individuals within the Hormathiidae. The categories, proposed by WEILL (1934), of microbasic and macrobasic mastigophores and amastigophores are substituted by the categories of $b$ - and p-rhabdoides. Within the Actiniaria p-rhabdoides differ from b-rhabdoides by having an unarmed thread, invaginated into the funnelshaped opening at the distal end of the uneverted shaft. The thread of b-rhabdoides, however, always bears spines. The shaft of some rhabdoides can be divided into a folded proximal part ("Faltstück") and a distal mainpart ("Hauptstück"). The two parts differ in structure and armature. These rhabdoides tend to throw out the mass of spines in a dartlike structure if discharge is hampered. Several new variations and categories of $b$ - and p-rhabdoides are presented; their taxonomic value is demonstrated by some examples.
\end{abstract}

\section{EINLEITUNG}

Der unterschiedliche Bau der Nesselkapseln der Cnidaria wurde schon früzeitig als taxonomisches Merkmal erachtet. MILNE EDwards (1857) berücksichtigte erstmals die Nesselkapseln bei der Beschreibung von Aktinienarten. Es folgten zahlreiche Veröffentlichungen, in deren Mittelpunkt die Morphologie und Funktion (Iwanzorf 1896, Will 1909, 1914, Cutress 1955, Hand 1961, Westrall 1964-1966, Skaer \& PrCKen 1965, Picken \& Skaer 1966) weniger aber die Bedeutung der Nesselkapseln. für die Systematik der Aktinien standen (Stephenson 1929, Carlgren 1940, 1945).

WeILL (1934) hat in einem umfassenden Werk die Nesselkapseln der verschiedenen Cnidarier in einem System vereint und eine umfangreiche Nomenklatur geschaffen. WeILls System wurde von allen Systematikern ubernommen, seine praktische 
Verwendung stieß jedoch auf Schwierigkeiten (RusseL 1953) und löste somit Kritik aus (Stephenson 1935, Carigren 1940, Cutress 1955). Bei der Charakterisierung seiner Nesselkapselkategorien wird WEnL in der Tat den taxonomischen Verhältnissen. der Aktinien und den morphologischen Gegebenheiten ihrer Nesselkapseln wenig gerecht. Dieser Mangel veranlaßte bereits Carlgren (1940) und Cutress (1955) zu weitreichenden Abänderungen einzelner Kategorien.

Heute ist WeILls System, obwohl erst 1965 von WERNER in den deutschen Sprachgebrauch eingeführt, nicht mehr mit den neuesten Ergebnissen (WESTFALL 1965, 1966, Picken \& SKaer 1966) in Einklang zu bringen.

Auf Grund eigener Untersuchungen an Aktinien sehe ich mich zu Abwandlungen des WerLschen Systems veranlaßt, soweit es die Nesselkapseln der Aktinien, insbesondere die rhabdoiden Heteronemen betrift.

\section{MATERIAL UND METHODE}

Nesselkapseln von 36 Aktinienarten aus dem Roten Meer, der Nordsee und dem Mittelmeer wurden in lebensfrischem Zustand im Dunkelfeld und im Phasenkontrast (nach Zernike) untersucht. Die Entladung der meisten Kapseln konnte mit Aqua destillata oder verdünntem Eisessig $(1: 10)$ erzielt werden.

Die elektronenmikroskopischen Untersuchungen fanden größtenteils an Totalpräparaten statt, ähnlich wie von RoBson (1953) beschrieben. Formvarbedeckte Kupfernetze wurden mit frischem Aktiniengewebe betupf, getrocknet und anschließend in verdünntem Eisessig oder Aqua destillata gebadet. Zur Herstellung von Ultradünnschnitten wurde das Material bei Zimmertemperatur in einem Gemisch aus 2,5\% Glutaraldehyd, 1,5\% NaCl und 0,13 M Phosphatpuffer ( $\mathrm{pH} 7,5) 12 \mathrm{~h}$ vorfixiert, anschließend in $1 \% \mathrm{OsO}_{4}$, gelöst in derselben Pufferlösung, $1 \mathrm{~h}$ nachfixiert und in Epon eingebettet. Kontrastiert wurde durch Zusatz von $1 \%$ Phosphorwolframsäure und $0,5 \%$ Uranylazetat zu $70 \%$ Alkohol.

\section{ERGEBNISSE}

Die Nesselkapseln der Aktinien bestehen aus „Spirocysten“ und „Nematocysten“. Der Nesselschlauch der Nematocysten kann bei einigen Kategorien in "Schaft“ (Achsenkörper) und „Faden“ unterteilt werden.

\section{Die Spirocysten}

Bis vor kurzem war umstritten, ob die Spirocysten gewöhnliche Nematocysten (Will 1909, Stephenson 1929, Cutress 1955) oder Nesselkapseln besonderer Art darstellen (WeILL 1934). Die jüngsten elektronenmikroskopischen Untersuchungen haben den besonderen Bau der Spirocysten offenbart. Neben der abweichenden Kapselbeschaffenheit hat WESTFALL $(1964,1965)$ als wesentliche Besonderheit unentladener Spirocysten festgestellt, daß der Fadeninhalt aus 30-40 parallel zur Längsachse des 

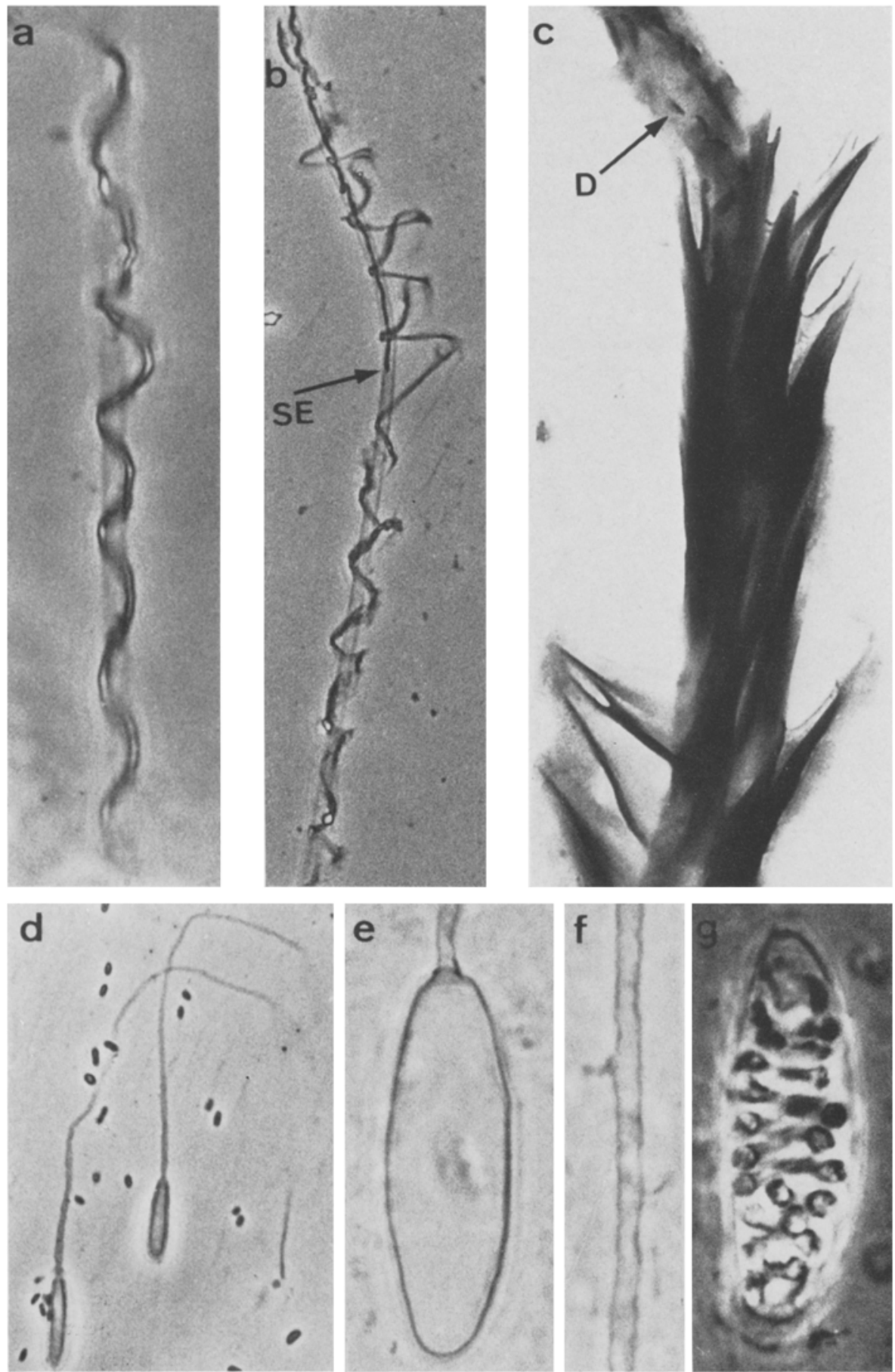
Fadens verlaufenden Stäbchen oder Tubuli besteht, deren Funktion sich die Autorin nicht erklären konnte.

Die eigenen Untersuchungen haben ergeben, daß die Spirocysten im Gegensatz zu den Beobachtungen von Cutress (1955) und HaND (1961) leicht zu entladen sind und in diesem Zustand deutlich von den Nematocysten abweichen. Analog zu den Nematocysten, deren Nesselschlauch über drei rechtsgewundene Dornspiralen verfügt, sollte nach bisheriger Auffassung der Schlauch der Spirocysten mit 3 quellfähigen Leisten versehen sein (WILL 1909, STEPHENSON 1929). Wie ich jedoch wiederholt an verschiedenen Aktinienarten beobachten konnte, windet sich um den unbewafineten Schlauch (SKaER \& PrCKeN 1965) der Spirocysten unmittelbar nach der Ausstülpung lediglich ein einziger Quellfaden, und zwar in Linkswindung (Abb. 1a, b). Dieser zunächst einheitliche Quellfaden ist nur locker mit dem Schlauch verbunden und löst sich in kurzer Zeit zu einem Sekretmaschenwerk auf, das auch schon von WILL (1909) und elektronenmikroskopisch von SKaER \& PICKEN (1965) dargestellt wurde.

Aus den elektronenmikroskopischen Aufnahmen von WeStFALL (1965, Pl. 6) geht hervor, daß die Tubulibïndel jeweils nur auf einer Seite des Schlauches zu sehen sind und das Schlauchinnere geradegestreckt durchziehen, ohne die Falten des Schlauches auszufüllen. Durch die Streckung dieser Falten jedoch erhält der Schlauch während der Entladung seine enorme Verlängerung. Der Quellfaden aber umgibt den Schlauch in weiten, lockeren Windungen und muß sich daher wesentlich länger als dieser entfalten können (Abb. 1b). Es liegt die Vermutung nahe, daß der Quellfaden im Inneren längs zur Schlauchrichtung gefaltet ist, so daß sich auf einem Schlauchquerschnitt immer mehrere Querschnitte des Quellfadens zeigen.

Die besondere Stellung der Spirocysten ist also gerechtfertigt, und ihr Bau läßt schwerlich einen Vergleich mit den übrigen Nematocysten zu, die ïber einen bewaffneten Schlauch ohne Quelleistungen verfügen.

\section{Die Nematocysten}

Nach WEILL (1934) bestehen die Nematocysten aus "Astomocniden" (ohne terminale Offnung) und "Stomocniden" (mit terminaler Offnung). Die Nematocysten der Aktinien gehören ausschließlich zu den Stomocniden, die wiederum in "Haplonemen" und "Heteronemen “ aufgegliedert sind (Tab. 1).

Diese Einteilung ist insofern unbefriedigend, als sich einerseits die Definitionen für den unentladenen Zustand überschneiden und andererseits der Kaliberunterschied von Schaft und Faden bei gewissen Heteronemen (b-Mastigophoren) so gering ist, daß er praktisch nur in wenigen Fällen feststellbar ist (CARLgren 1940, CuTress 1955, HaND 1961).

Abb. 1: (a) Spirocyste, Alicia costae, Capitulum, Schlauch mit Quellfaden (1500:1); (b) Spirocyste, $A$. costae, Quellfaden aufgelockert, Schlauchende (SE) noch nicht entladen (1050:1); (c) b-Rhabdoide, Bunodactis verrucosa, Mesenterialfilament: Schaft und Faden, Faden mit Dornen (D) $(18000: 1)$; (d) Anisorhize Haploneme, Aiptasia diapbana, Scapus $(1100: 1)$; (e-g) Isorhize Haploneme, Sagartiogeton undatus, Scapus: entladen (e), Faden (f), geschlossen $(\mathrm{g})(1890: 1)$ 
Tabelle 1

Unterscheidung der beiden Kategorien von Stomocniden (vgl. WERnER 1965)

\begin{tabular}{|c|c|c|}
\hline Stomocniden & Entladen & Nicht entladen \\
\hline Haplonemen & $\begin{array}{l}\text { Nesselapparat einfacher } \\
\text { Faden, ohne deutlich ab- } \\
\text { gesetzten basalen Schaft. }\end{array}$ & $\begin{array}{l}\text { Ohne Achsenkörper oder } \\
\text { mit schwach entwickeltem } \\
\text { Achsenkörper. }\end{array}$ \\
\hline Heteronemen & $\begin{array}{l}\text { Nesselapparat mit deutlich } \\
\text { abgesetztem basalem Schaft } \\
\text { oder Schaft ohne Faden. }\end{array}$ & $\begin{array}{l}\text { Mit schwach oder stark } \\
\text { entwickeltem Achsenkörper. }\end{array}$ \\
\hline
\end{tabular}

Es bestehen jedoch enge Beziehungen zwischen der Bewaffnung und der Gestalt des Nesselschlauches, die geeigneter sind, die Haplonemen von den Heteronemen $z u$ unterscheiden. Einem schwach oder stark entwickelten Achsenkörper einer unentladenen Stomocnide entspricht in entladenem Zustand weniger ein deutlich abgesetzter basaler Schaft als vielmehr eine deutlich abgesetzte Bewaffnung. Nach dieser Definition zeichnen sich also die Heteronemen durch eine langbedornte Basis (Schaft) aus, an die sich ohne Ubergang ein kurz oder nicht bedornter Faden anschließt, und zwar unabhängig davon, ob sich das Kaliber des Nesselschlauches an irgendeiner Stelle abrupt ändert oder nicht (Abb. 2c, d).

Bei den Haplonemen hingegen besteht entweder kein bedeutender Unterschied in der Bewaffnung der einzelnen. Schlauchabschnitte ("Isorhizen") oder aber der Nesselschlauch verjüngt sich distal auffällig und die Dornen nehmen entsprechend dem jeweiligen Schlauchquerschnitt an Länge ab („Anisorhizen“). Daraus folgt, daß die Haplonemen keinen Achsenkörper im Sinne Werlss (1934, p. 53) haben können. Ihr Schlauch ist gleichmäßig oder ungleichmäßig in der Kapsel aufgerollt (Abb. 2a, b).

\section{Die Haplonemen}

Eine Unterscheidung von isorhizen und anisorhizen Haplonemen erscheint nach den ausführlichen Untersuchungen an holotrichen Haplonemen nicht mehr angebracht. Skaer \& PiCken (1965) haben für WeILls Typus einer holotrichen isorhizen Haploneme von Corynactis viridis (Corallimorpharia) an elektronenmikroskopischen Präparaten nachgewiesen, daß es sich bei dieser Stomocnide um eine heterotriche anisorhize Haploneme handelt.

Obwohl sich vielleicht bei elektronenmikroskopischer Betrachtung letztlich alle Haplonemen als Anisorhize herausstellen werden, sollte jedoch aus praktischen Gründen diese Unterteilung beibehalten werden. Denn bei lichtoptischer Untersuchung liefert diese Differenzierung wertvolle Anhaltspunkte für die Systematik. Die Aufteilung der Haplonemen durch WEILL (1934) in: (1) atriche, basitriche und holotriche Isorhizen ${ }^{1}$ und (2) homotriche und heterotriche Anisorhizen ist nicht gerechtfertigt.

1 WeRnER (1965) schuf für die Hydrozoen den neuen Typ der merotrichen Isorhizen, die über einen der Basis genäherten $Z$ wischenteil mit abgesetzter Bewaffnung verfügen. Es handelt sich laut vorliegender Definition um eine Heteroneme. 
Alle näher untersuchten "Atrichen" erwiesen sich als "holotrich" (Curress 1955, Picken \& Skaer 1966, Westfall 1965). Die Dornen der ehemaligen „Atrichen“ unterscheiden sich nicht von denjenigen der "Holotrichen" (PICKEN \& SKaER 1966). "Basitriche" im Sinne WeIlls existieren nicht. Sie sind identisch mit Carlgrens

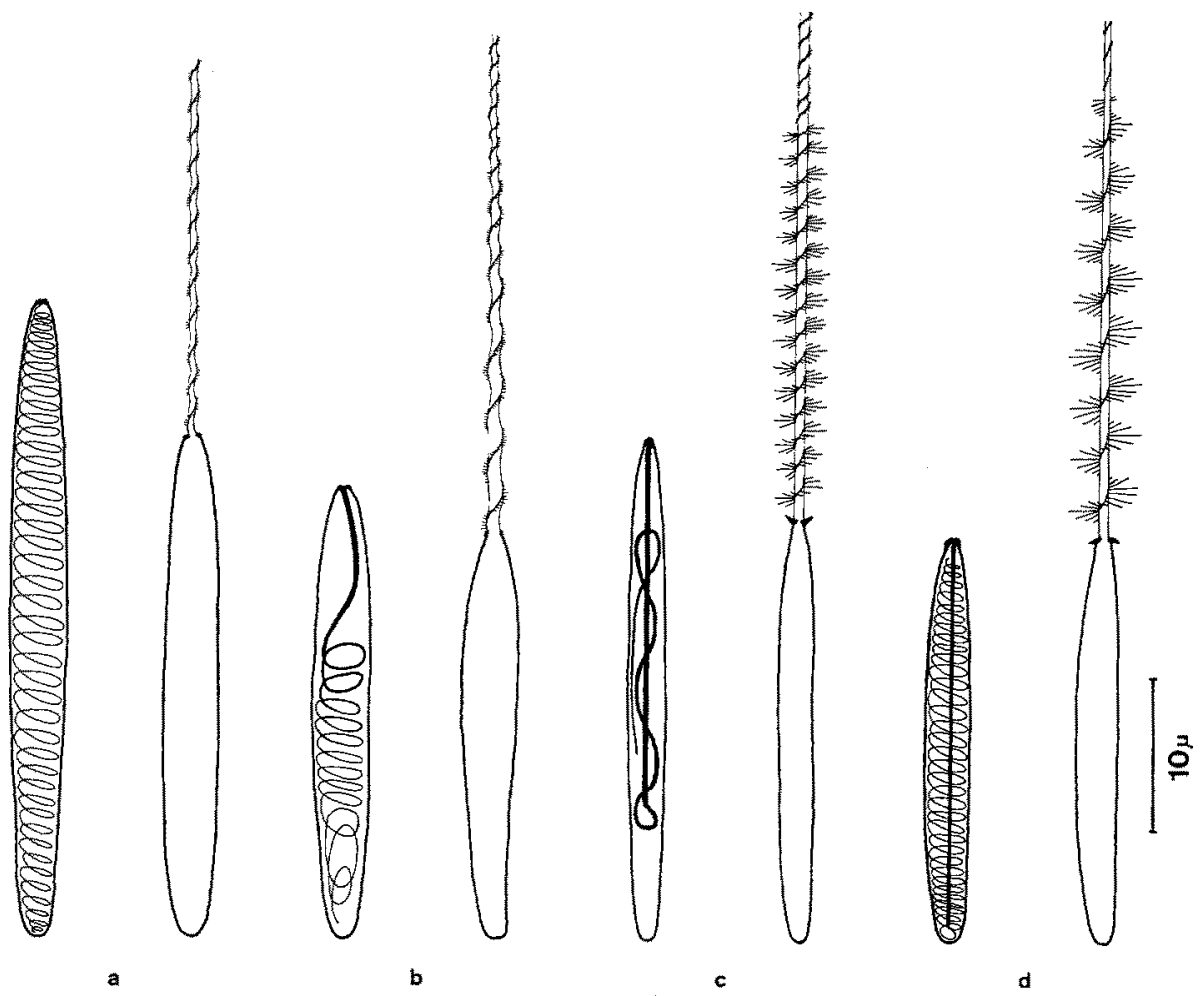

Abb. 2: (a) Isorhize Haploneme, Anthopleura stellula, Akrorhage; (b) Anisorhize Haploneme, Antbopleura rubripunctata, Akrorhage; (c) b-Rhabdoide, Aiptasiogeton (Sagartiogeton) laceratus, Akontium: Faden $3 \times$ Schaft; (d) b-Rhabdoide, Sagartiogeton undatus, Akontium: Faden $16 \times$ Schaft

„b-Mastigophoren“ (Cutress 1955, WestFali 1965, 1966a, 1966b, vgl. Carlgren 1940). Die von Cutress neu definierten „Basitrichen" sind nach meinen Untersuchungen an Aktinien der gleichen Gattungen, die Cutress (1955) als Beispiele anführt, entweder b-Mastigophoren oder Haplonemen.

Anisorhize Haplonemen waren bisher bei den Aktinien nicht bekannt. In verschiedenen Arten fand ich jedoch heterotriche Anisorhizen in sehr ausgeprägter Form. Homotriche Anisorhizen kommen dagegen nicht vor. Da der Schlauch der Haplonemen im ibrigen sehr wenig differenziert ist, liegt die taxonomische Bedeutung mehr in der Formenmannigfaltigkeit. So ist zum Beispiel bei großen dünnwandigen Haplonemen der Schlauch in der Kapsel besonders gut von dem stark kontrastierenden Kapselinhalt zu unterscheiden. Derartige Formen verfügen meist über einen langen weitgehend iso- 
diametrischen Schlauch (Abb. 1e-g). Andere Haplonemen besitzen einen so kontrastreichen Inhalt, daß die Untersuchung des Schlauchs im Kapselinnern kaum möglich ist. Es handelt sich zumeist um dickwandige Anisorhize (Abb. 1d). Zwischen diesen beiden Nesselkapseln gibt es alle möglichen Ubergangsformen.

Manche Haplonemen treten nur sehr sporadisch auf. Wie bekannt (CARLGren 1929), ist der Besitz von Haplonemen bei einigen Arten an die Ausbildung von Fangtentakeln gebunden, die nur wenigen Individuen eigen sind. Bei Calliactis parasitica und bei Amphianthus dorni beobachtete ich dagegen bei einzelnen Exemplaren massenhaft Haplonemen an allen Tentakeln, ohne daß diese morphologische Abweichungen aufwiesen. Die Haplonemen sind bisher nur aus Scapus und Tentakeln bekannt. Bei Sagartia troglodytes fand ich sie auch in den Akontien. Die von BonNenfant (1960) in den Mesenterialfilamenten von Actinia equina beschriebenen Atrichen und Spirocysten sind b-Mastigophoren. Die Autorin hat wiederholt Haplonemen mit diesem Nesselkapseltypus verwechselt, wie ich auch für Aulactinia crassa nachweisen konnte (SсHмidT 1967).

Im Gegensatz zu den Spirocysten sind die Haplonemen basophil und zeichnen sich durch einen deutlichen Phasenkontrast aus.

\section{Die Heteronemen}

\section{Die gegenwärtige Klassifizierung}

Von den drei Unterordnungen der Heteronemen (vgl. WeRner 1965, Tab. 1, p. 8) sind lediglich die Rhabdoiden bei den Aktinien vertreten. WeILL (1934) unterteilte sie in mikrobasische (Schaft bis zu dreifacher Kapsellänge) und makrobasische (Schaft vierfache Kapsellänge und länger) Mastigophoren bzw. Amastigophoren. Von diesen sollen den Aktinien die makrobasischen Mastigophoren fehlen.

In den Amastigophoren schuf WeILL einen Typ, der sich nicht nur von allen anderen Heteronemen durch das Fehlen des Endfadens unterscheidet (WeILL 1934, p. 68), sondern auch in seinem Vorkommen allein auf eine einzige Ordnung innerhalb der Cnidaria beschränkt ist, nämlich auf die Aktinien (WERNER 1965, Tab. 2, 5 und 6). Die Heteronemen der Aktinien waren also bestimmend für die Einteilung der Rhabdoiden, obwohl WeILl, wie auch CARLgReN (1940, p. 5) bemerkte, nur relativ wenig Aktinienmaterial untersudat hat. Dies mag der Grund dafür sein, daß die Nesselkapselkategorien der Aktinien in der Folgezeit häufige Abänderungen erfahren haben.

CARLgREN (1940) unterteilte die mikrobasischen Mastigophoren in „p-Mastigophoren" mit einer trichterförmigen Einstülpung am distalen Schaftende der unentladenen Kapsel, und in "b-Mastigophoren", deren Schaft sich in unentladenem Zustand plötzlich zu einem dünnen Faden verjüngt.

Die Entdeckung eines Fadens bei unentladenen Amastigophoren war für CuTress (1955) ausreichender Grund, die Eliminierung der Amastigophoren vorzuschlagen, zumal er gelegentlich sogar entladene Amastigophoren mit ausgestülptem Faden beobachtet hatte. Als zusätzliche Kategorien führte Cutress die "mikrobasischen q-Mastigophoren" und die "makrobasischen p-Mastigophoren" ein. 
Wesentlicher Bestandteil der q-Mastigophoren ist ein pfeilförmiges Gebilde, das dem eigentlichen Schaft lose aufsitzt und sich während der Entladung von dem Schaft trennt.

In der Kategorie der makrobasischen p-Mastigophoren faßte Cutress die makrobasischen Amastigophoren verschiedener Aktinien, die b-Mastigophoren der Ceriantharien und die Holotrichen der Corallimorphen zusammen, da diese Nesselkapseltypen im Gegensatz zu ihren herkömmlichen Kategorien ebenso wie die mikrobasischen p- und q-Mastigophoren über einen in sich selbst noch einmal invaginierten Schaft verfügen sollen, der von einem Faden der Länge nach durchlaufen wird. Inzwischen ist jedoch durch elektronenmikroskopische Untersuchungen bekannt, daß sich der Schaft bei den Holotrichen der Corallimorphen (SKAer \& PICKEN 1965) und bei den mikrobasischen Amastigophoren der Aktinien (WESTFALL 1962) nur einfach in die Kapsel eingestülpt. Schließlich konnte WESTFALL (1965) im Elektronenmikroskop bestätigen, daß auch bei den Amastigophoren wie bei den Mastigophoren aus der trichterförmigen Einstülpung des distalen Schaftendes in der unentladenen Kapsel ein Faden ragt, dessen Verbindung mit dem Schaft jedoch unentdeckt blieb.

\section{Befunde}

(1) Bei meinen Untersuchungen konnte ich wiederholt die Feststellung machen, daß bei Metridium, Bunodeopsis, Alicia, Diadumene, Boloceroides, verschiedenen Sagartiiden und Aiptasiiden das Fehlen des Fadens nach der Entladung durchaus keine konstante Erscheinung ist. Im gleichen Präparat besaß ein Teil der Amastigophoren am Ende ihres Schaftes einen ausgestülpten Faden, der häufig sogar die Länge der Kapsel erreichte. An einigen Amastigophoren konstatierte ich regelmäßig ausgestülpte lange Fäden. Andererseits blieb der Faden bei meinen Beobachtungen an Telmatactis elongata nach der Entladung konstant in der Kapsel zurück. Der Faden ist auch in der Kapsel in jedem Fall sichtbar (Abb. 7g). Im Elektronenmikroskop konnte ich an explodierten Amastigophoren verschiedener Aktinien erkennen, daß der Faden mit einem Ende nicht an der Kapselwand befestigt ist, wie Cutress (1955) vermutete, sondern frei in leicht gedehntem Zustand in der Kapsel liegt.

Diese Befunde zeigen, daß die Amastigophoren im Sinne WeILls (1934, p. 68) für die Systematik nicht die Bedeutung besitzen, die WEILL ihnen in seinem System einräumt. Bei der Einteilung der Rhabdoiden sollten sie daher nur eine untergeordnete Rolle spielen, zumal noch nicht erwiesen ist, inwieweit sich die jeweilige Untersuchungstechnik auf die Ausstïlpung des Fadens auswirkt.

(2) Makrobasische Amastigophoren zeichnen sich nach WEILL (1934, Fig. 62) nicht nur durch einen sehr langen Schaft und das Fehlen des Fadens, sondern auch durch eine besondere Bewaffnung aus. Der Typ ist nur von einigen Aliciiden und von Diadumene (CARLGREN 1940, HAND 1955) und als makrobasische p-Mastigophore von Bunodeopsis bekannt (CuTRESS 1955). CARLGREN (1940) betrachtete zwar bestimmte mikrobasische Amastigophoren von Boloceroides und Bunodeopsis als Übergangsstadien zu WeILls makrobasischen Amastigophoren, hatte aber andererseits die große Ahnlichkeit gewisser mikrobasischer Amastigophoren aus den Blasen und Tentakeln 
von Bunodeopsis strumosa mit den makrobasischen Amastigophoren der Aliciiden nicht erkannt (CARLGREN 1925, 1940). Bei Boloceroides und Bunodeopsis haben die betreffenden mikrobasischen Amastigophoren jedoch die gleiche Bewaffnung wie WeILLs makrobasische Amastigophoren (Abb. 3d). Außerdem wird in beiden Fällen ein kurzer Faden ausgestülpt. Bei Boloceroides me murrichi hat dagegen der Schaft nur etwa die
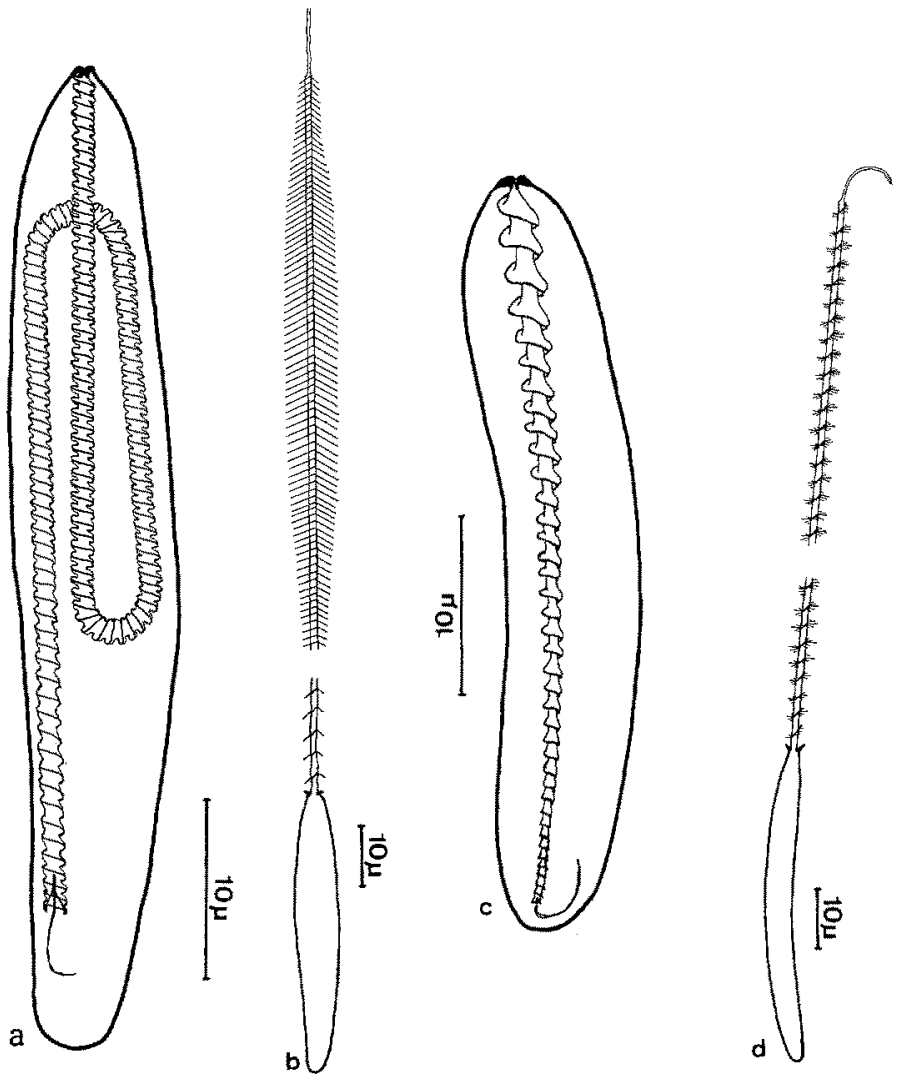

Abb. 3: (a) p-Rhabdoide B 2b, Diadumene cincta, Tentakel; (b) p-Rhabdoide B 2b, D. cincta, Schaft insgesamt 7,25 $\times$ Kapsel, Faltstïck 2,5 $\times$ Hauptstïck; (c) p-Rhabdoide C, Bunodeopsis strumosa, Scapus; (d) p-Rhabdoide C, Boloceroides mc murrichi, Tentakel

dreifache und bei Bunodeopsis strumosa sogar nur die doppelte bis dreifache Kapsellänge. Die makrobasischen Amastigophoren aus den Tentakeln von Diadumene sind noch nicht in entladenem Zustand beobachtet worden. Die Bewaffnung dieser Nesselkapsel gleicht nämlich derjenigen der meisten mikrobasischen $\mathrm{p}$-Mastigophoren und mikrobasischen Amastigophoren (Abb. 3b). Die Nesselkapsel stülpt außerdem stets einen kurzen Faden aus.

Die Befunde zeigen, daß WEILls Begriffe "mikrobasisch" und "makrobasisch" zur Einteilung der Rhabdoiden ungeeignet sind, nämlich insofern als durch diese Unter- 
teilung morphologisch gleichartige Nesselkapseln zwei verschiedenen Kategorien zugeordnet werden. Die Schaftlänge eignet sich also ebensowenig wie die Ausstülpung des Fadens als Merkmal für die Klassifizierung der rhabdoiden Heteronemen.

(3) Während der Reifungsphase vollzieht sich die Ausbildung von Schatt und Faden bei den Amastigophoren in derselben Weise wie bei den p-Mastigophoren. Wie schon IWANZOFF (1896) beobachtete, nehmen die Nesselkapseln der Aktinien während der Reifung erheblich an Volumen $a b$. Die Kapsel verringert zuerst ihre Länge und dann ihre Breite (Abb. 10a). Mit der Volumenabnahme vollzieht sich gleichzeitig eine
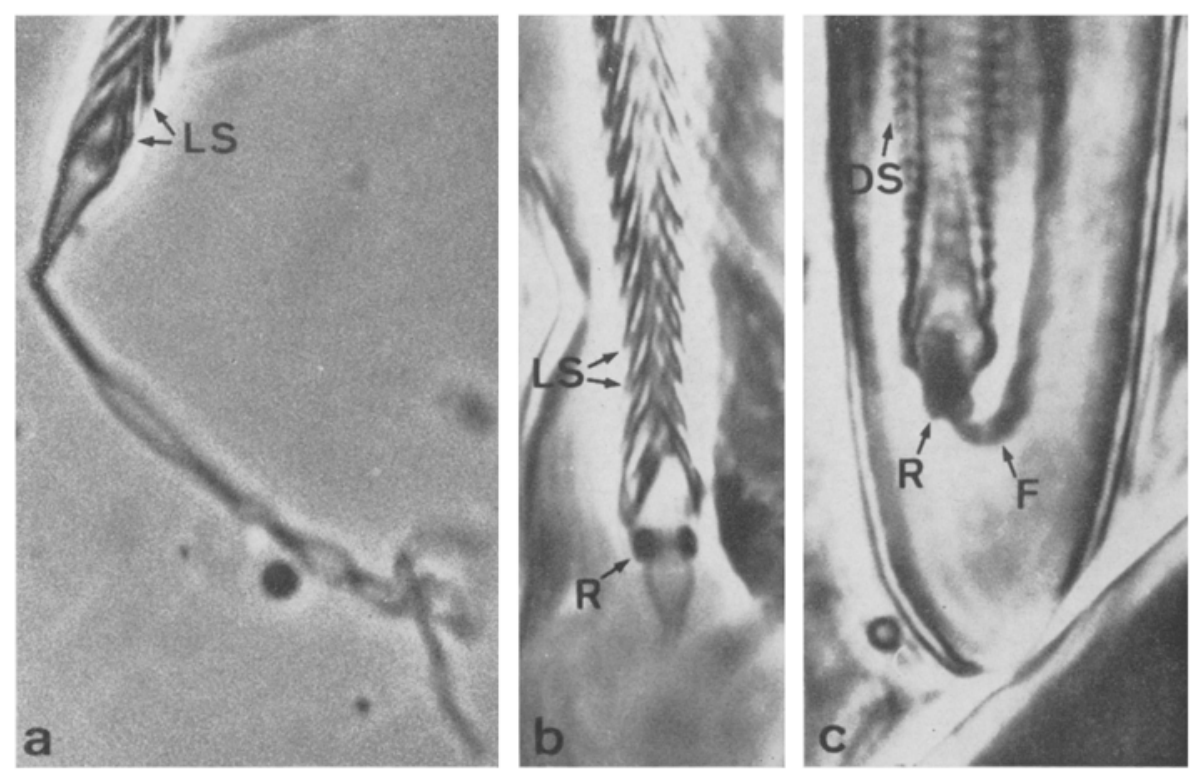

Abb. 4: (a) p-Rhabdoide B 2a, Aiptasia conchii, Akontium: unreifer Schaft und Faden mit lockerer Schraubenstruktur (LS) (3000:1); (b) p-Rhabdoide B 1b, Telmatactis elongata, Akontium: unreifer Schaft und Faden mit lockerer Schraubenstruktur (LS) und ringförmiger Wandverdickung (R) zwischen Schaft und Faden $(3500: 1)$; (c) p-Rhabdoide B 2a, Aiptasia coucbii, Akontium: fast reife Nesselkapseln, kontrahierter Schaft mit dichten Schraubenwindungen (DS), Einstülpung des Fadens (F) durch die ringförmige Wandverdickung (R) (3900:1)

Kontraktion des Nesselapparates. Vor der Kontraktion liegen Schaft und Faden in aufgelockertem Zustand in der Kapsel (Abb. 4a). Dabei ist festzustellen, daß der Faden die natürliche Fortsetzung der Schaftwandung ist, so wie es von den b-Mastigophoren auch in reifem Zustand bekannt ist (CARLGREN 1940, HaND 1961). Kurz vor der Kontraktion entsteht $z$ wischen Schaft und Faden eine ringförmige Wandverdikkung (Abb. 4b). An dieser Stelle stülpt sich der Faden am Ende der Kontraktionsphase in die trichterförmige distale Schaftöffnung ein (Abb. $4 c$ ). In reifem Zustand entspricht dieser Abschnitt der Basis des konusförmigen Ubergangsteiles zwischen Schaft und Faden. Die Wandung dieses konusförmigen Übergangsteiles ist auch in entladenem Zustand geringfügig dicker als die des Fadens (vgl. Westrall 1965, Pl. 5, Fig. 11).

Der verschiedenartige Fadenansatz ist eine konstante Erscheinung und ein deut- 
liches Unterscheidungsmerkmal für zwei Nesselkapseltypen, die ich im folgenden in Anlehnung an CARLGREN (1940) „b-und p-Rhabdoiden" nenne.

(4) Nach Iwanzorf (1896) besteht der ausgestülpte Schaft der p-Rhabdoiden der Akontien von Aiptasia diaphana aus einem mit kürzeren Dornen und „zusammengeschrumpften "Wänden versehenen proximalen und einem langbedornten glattwandigen distalen Teil (Abb. 7h, 9a). Ich nenne den proximalen Teil „Faltstück" und den distalen Teil "Hauptstiick". Das Hauptstïck ist wie ein Schaft ohne Faltstück anisodiametrisch (vgl. Abb. 8). Mit abnehmendem Querschnitt werden distal die Dornen kleiner, ohne jedoch dabei von ihrer Grundform abzuweichen (Abb. 9f). Dieser Befund stimmt mit den Beobachtungen von SKAER \& PICKEN (1965) an Haplonemen von Corynactis viridis überein. Die Dornenwindungen haben jedoch am distalen Schaftende und an dem ebenfalls schmaleren Faltstück einen größeren Abstand als diejenigen des übrigen Schaftes (Abb. 9a). Die Dornen des Faltstückes unterscheiden sich aber von denjenigen des Hauptstïckes insofern, als sie etwa nur halb so breit sind wie vergleichbare Dornen des distalen Hauptstückes ( $A b b, 9 b, c, f)$. Die besonders breite Basis dient einer scharnierförmigen Verankerung an den Falten des Faltstückes (Abb. 9d). Eine ähnliche Befestigung der Dornen hatten Skaer \& Picken (1965) an unentladenen Haplonemen beobachtet.

In unentladenem Zustand besitzt der Schaft eine dreifache linksgedrehte Schraubenstruktur (Bretschneider 1949, Picken \& SkAer 1966). Der Abstand der einzelnen Schraubenwindungen variiert (Abb, 5). Er ist im Gegensatz zu den entsprechenden Dornenwindungen im entladenen Zustand in der Faltstückregion und am distalen Schaftende dichter als in der Mitte.

Die Dornen richten in der geschlossenen Kapsel ihre Spitzen gegen die Kapselöffnung und bilden in ihrer Gesamtheit den pfeilförmigen stark kontrastierenden Inhalt des Schaftes (Abb. 5, 7c, 6a). In der Faltstückregion rücken die Dornen infolge der stark gefurchten, dichten Schraubenwindungen in die Mitte des Schaftes und sind infolgedessen wesentlich steiler angeordnet als im Hauptstïck. Die steile Stellung und die dichten Schraubenwindungen bedingen die scharnierförmige Verankerung und die besondere Form der Dornen des Faltstïckes (Abb. 9b, c). Am distalen Schaftende sind die Dornen, bedingt durch die trichterförmige Einstülpung und die dichteren, aber weniger stark gefurchten Schraubenwindungen, lediglich kleiner (Abb. 9f).

WEILL (1934, p. 63) vertritt den Standpunkt, daß alle Rhabdoiden ein Faltstück besäßen. Nach meinen Beobachtungen jedoch fehlt einem Teil der Rhabdoiden ein derartiges Faltstück (Abb. 7e-h).

(5) Durch die Schraubenstruktur erhält der Schaft eine gewisse Elastizität, die besonders groß im proximalen Faltstück ist, da hier keine oder nur wenige kurze Dornen liegen. Dieser Umstand führt bei manchen Rhabdoiden zur Entstehung von pfeilförmigen Gebilden während der Entladung (Abb. 6c). Derartige Pfeile wurden schon von IWANZOFF (1896) und WEILL (1934) als eine Zusammenballung einzelner Dornen erkannt. Cutress (1955) jedoch betrachtete diese Gebilde als typisches Merkmal einer neuen Nesselkapselkategorie, der "mikrobasischen q-Mastigophoren “. Er hat allerdings übersehen, daß WEILL (1934) Pfeile an zwei verschiedenen Nesselkapselkategorien aus den Akontien von Metridium senile beobachtet hatte, nämlich an den p-Rhabdoiden (von WEIL 1934 mikrobasische Amastigophoren genannt, p. 144, Fig. 
121, 122) und an den b-Rhabdoiden (von Werll in diesem Fall mikrobasische Mastigophoren genannt, p. 144, Fig. 123-125). Bei der Untersuchung derselben Nesselkapseltypen aus den Akontien der gleichen Art konnte ich die Entstehung der Pfeile an den b-Rhabdoiden besonders gut beobachten (Abb. 6a-c). Dieser Nesselkapseltyp verfügt

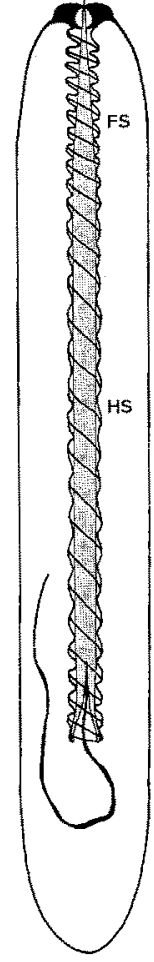

Abb. 5

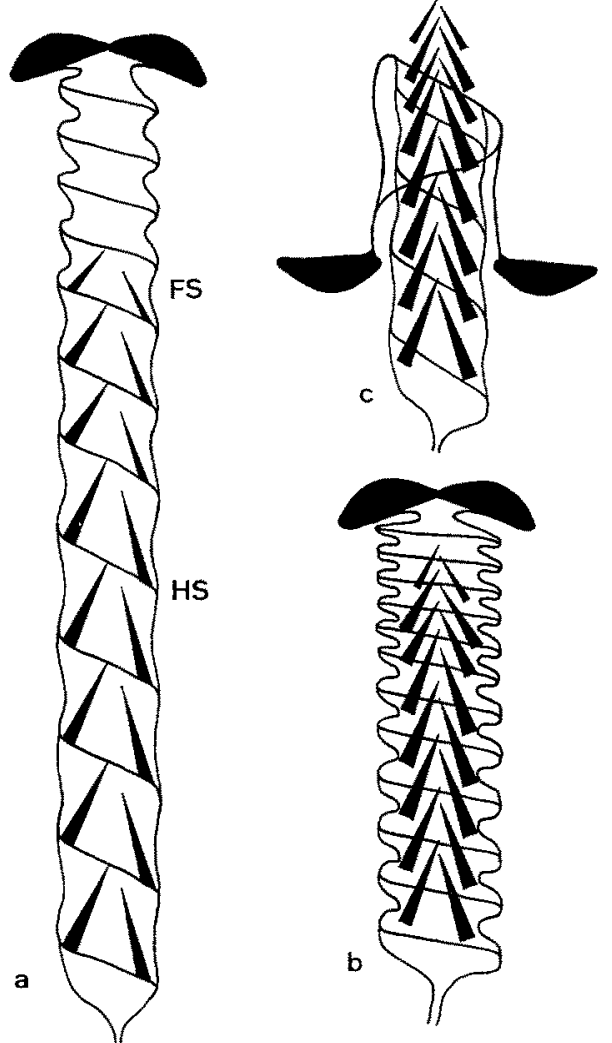

Abb. 6

Abb. 5: p-Rhabdoide B 2a, Modell: Hauptstück (HS), Faltstück (FS)

Abb. 6: (a) b-Rhabdoide, Schaftmodell, Metridium senile, Akontium: Schaft nicht entladen, Hauptstück (HS), Faltstück (FS); (b) Schaft vor der Entladung in der Längsrichtung gestaucht, Dornen aus der Verankerung gelöst und zusammengedrängt; (c) Schaft während der Entladung, Auswurf des „Pfeiles"

ebenfalls über ein Faltstück, das an seinem proximalen Teil keine Dornen trägt (vgl. Westfall 1965, P1. 2, Fig. 2), dann aber bewaffnet ist und allmählich in das länger bedornte Hauptstück übergeht (Abb, 6a).

$\mathrm{Zu}$ Beginn der Entladung wird der Schaft bei allen Rhabdoiden unter hohem Druck blitzartig ausgestülpt. Wird die Evagination aus irgendeinem Grund verzögert, so drückt der hohe Innendruck der Kapsel den Schaft in seiner Längsrichtung zusammen (Abb. 6b). Bei den b-Rhabdoiden sind die Dornen weniger dicht gelagert als bei den p-Rhabdoiden (vgl. Westrall 1965, Pl. 2, 5). Das hat zur Folge, daß sich der 

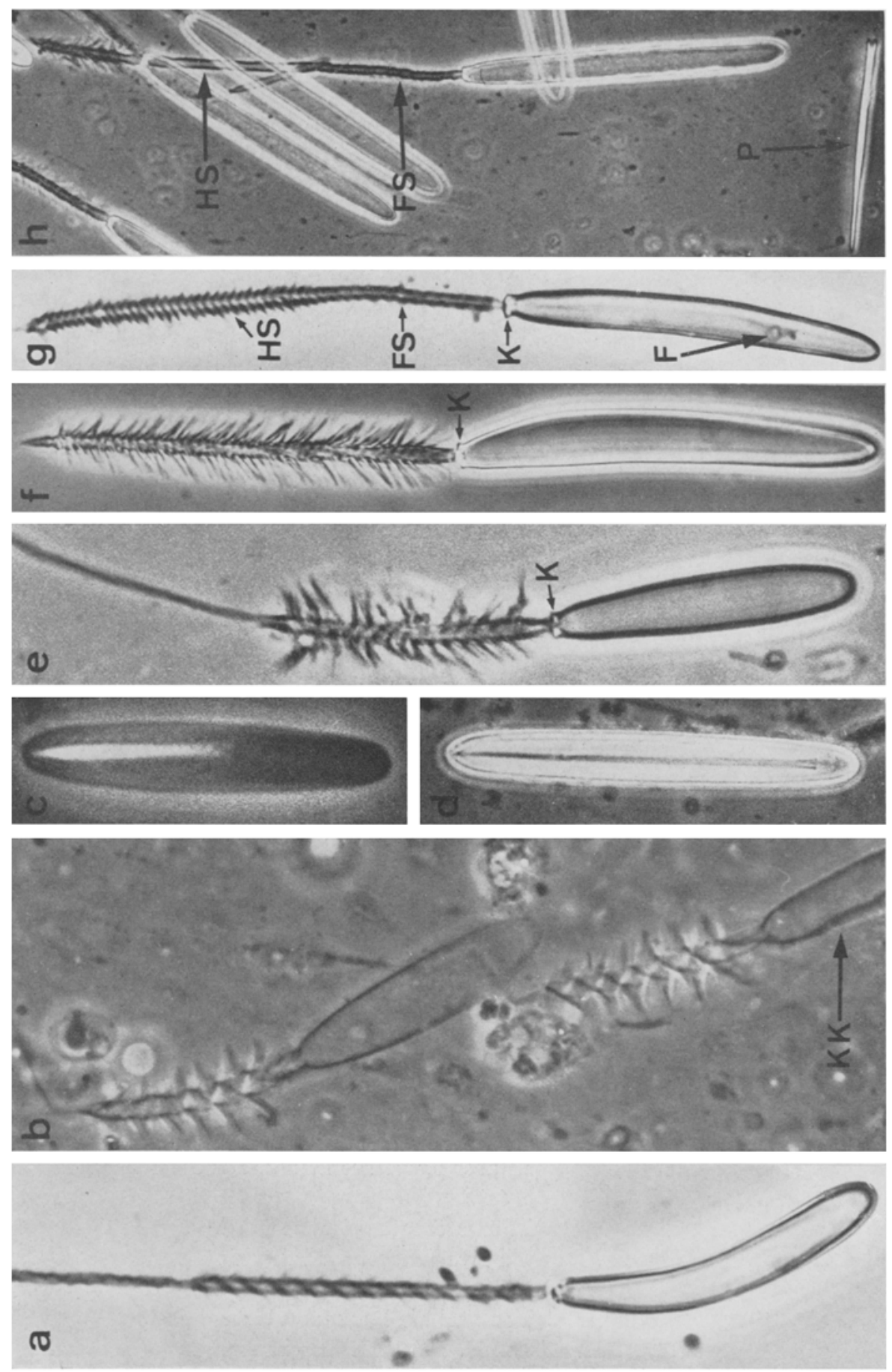
Schaft unter Druck bis auf die Hälfte seiner ursprïnglichen Länge verkürzen kann oder sogar verknäuelt (vgl. WEILL 1934, p. 61, Fig. 53c). Dadurch werden die Dornen von der stark gefalteten Schaftwand gelöst und an der Stelle, an welcher der Druck von beiden Seiten gleich groß ist, zusammengedrängt. Nach beendeter Evagination umgürten dann die Dornen unter Umständen in Form eines Pfeiles oder mehrerer Pfeile den Schaft in rechtsgewundenen Spiralen (vgl. Werl 1934, p. 144, Fig. 123). Entsprechend der geringen Dichte der Bewaffnung ist der Pfeil der b-Rhabdoiden ein wesentlich lockereres Gebilde als bei den p-Rhabdoiden und zerfällt daher häufig in einzelne Bruckstücke, die, durch die Anordnung der Dornen bedingt, wiederum Pfeilform besitzen. Die Bildung von mehreren Pfeilen durch eine einzige Nesselkapsel ist jedoch von Cutress (1955) ausdrücklich in Abrede gestellt worden.

Bei den p-Rhabdoiden wird in erster Linie das elastischere Faltstück gestaucht. Dabei werden die Dornen insgesamt oder teilweise aus ihrer Verankerung gelöst und zur Kapselöffnung hin verschoben, da sie so dicht gelagert sind, daß sie kaum mehr zusammengedrückt werden können (vgl. HAND 1961). Auf diese Weise wird der stark kontrastierende pfeilförmige Schaftinhalt während der weiteren Evagination im Zusammenhang aus dem Schaft ausgeworfen und gleicht daher in seiner Form demjenigen des unentladenen Schaftes (Abb. 5, 7h). Da sich der Druck von proximal nach distal fortpllanzt, ist häufig nach der Evagination nur noch das distale Schaftende bewaffnet. Je länger der Pfeil ist, desto geringer ist der Schaft bewaffnet.

Die Behinderung der Schaftausstülpung liegt häufig an dem Kapselverschluß, der aus drei Klappen besteht (WestFall 1962). Die Klappen werden bei der Entladung um $180^{\circ}$ gedreht. Dabei bilden die langen Klappenfortsätze mancher Rhabdoiden wahrscheinlich ein besonderes Hindernis (Abb. 9d). Die Schaftausstiilpung kann jedoch auch während der Evagination an jeder beliebigen Stelle behindert sein, so daß Form und Länge der Pfeile sehr unterschiedlich ausfallen können. Bei kleineren Pfeilen fällt die mangelhafte Bewaffnung nicht auf (vgl. Cutress 1955). Bei größeren Pfeilen kann der gesamte Schaft unbewaffnet sein (vgl. Hand 1961). Mitunter wird ein Teil der Dornen in die Falten des Faltstïckes gepreßt, so daß auch bei den p-Rhabdoiden der Pfeil aus mehreren Stücken bestehen kann.

Ich habe die Pfeilbildung bei verschiedenen Aktinienarten beobachtet, besonders häufig bei Metridium, Aiptasia und Aiptasiogeton (Sagartiogeton) laceratus, nicht hingegen bei Diadumene (vgl. Hand 1961). Die Pfeilbildung ist keineswegs ein konstantes Merkmal einer bestimmten Nesselkapselkategorie (CuTress 1955). Vielmehr gibt es gewisse Nesselkapseln mit mehr oder weniger großer Disposition, Pfeile zu bilden. Viele dieser Nesselkapseln entladen sich auch ohne Pfeilbildung. Prädestiniert für die

Abb. 7: (a) b-Rhabdoide, Parantbus chromatoderus, Scapus (2180:1); (b) p-Rhabdoide A, Tealia felina, Mesenterialfilament: Kapsel kollabiert (KK) $(1500: 1)$; (c) p-Rhabdoide A, T. felina (1920:1); (d) p-Rhabdoide B 2a, Aiptasia mutabilis, Akontium (620:1); (e) pRhabdoide B 1a, Paractinia striata, Mesenterialfilament: Schaft ohne Faltstiick, Kapselöfnung mit Klappen (K) (1830:1); (f) p-Rhabdoide B 1b, Telmatactis elongata, Akontium; Schaft ohne Faltstick, Klappen (K) $(815: 1)$; (g) p-Rhabdoide B $2 a$, Cerews pedunculatus, Akontium: Hauptstück (HS), Faltstïck (FS), Klappen (K), Faden (F) in der Kapsel (fokusiert auf Dornenwindungen und Faden) (1090:1); (h) p-Rhabdoide B 2a, Aiptasia mutabilis, Akontium: Faltstiuck (FS) und proximaler Teil des Hauptstückes (HS) durch "Pfeilbildung" unbewaffnet, zugehöriger "Pfeil" (P) (535:1) 
Pfeilbildung sind solche Nesselkapseln, die über einen geradlinigen Schaft mit elastischem Faltstück verfügen. Bei Nesselkapseln ohne Faltstuick ist die Pfeilbildung nur sehr selten zu beobachten. Allein auf Grund dieser Erscheinung ist es nicht möglich, eine neue Kategorie zu errichten, da Pfeile bei so unterschiedlichen Typen wie den b- und p-Rhabdoiden auftreten. Bei einigen Aktinienarten kommen allerdings in den Akontien gehäuft Pfeile vor, deshalb sollten sie nicht unerwähnt bleiben.

\section{Die Neugruppierung der rbabdoiden Heteronemen}

Der stabförmige Schaft kennzeichnet die Rhabdoiden in genügendem Maße. Obwohl keineswegs isodiametrisch (Definition von WeILl 1934, p. 38), fehlen dem Schaft rundliche Anschwellungen, die für die übrigen Heteronemenkategorien typisch sind. Wie die Befunde zeigen, müssen für die Neueinteilung der Rhabdoiden andere Kriterien herangezogen werden.

In einer Anmerkung zu seinem System erklärt WEILI, daß alle Heteronemenkategorien mit Ausnahmen der Amastigophoren nochmals in "Hoplotele" und "Anoplotele" unterteilt werden können, je nachdem, ob der Faden bewaffnet oder unbewaffnet ist. Von dieser Klassifizierungsmöglichkeit wurde aber kein Gebrauch gemacht. Curress (1955) schlug vor, alle b-Rhabdoiden als hoplotel zu betrachten und diesem Merkmal wenig Bedeutung zukommen zu lassen. WeILl (1934) hatte dagegen bei den Aktinien nur anoplotele Rhabdoiden beschrieben. Es waren jedoch berechtigte Zweifel an WExLs Aussage hinsichtlich der Bewaffnung des Fadens entstanden, als WESTFALL (1965) elektronenmikroskopisch an Metridium senile feststellte, daß die Basitrichen aus den Tentakeln gleichermaßen wie die b-Mastigophoren aus den Akontien - beide Nesselkapseln wurden von WerLL (1934, p. 58, Fig. 44; p. 63, Fig. 53) als anoplotele mikrobasische Mastigophoren eingestuft - einen bedornten Faden aufweisen.

An entladenen Rhabdoiden der untersuditen Aktinienarten habe ich festgestellt, daß der Faden auch der kleinsten $b$-Rhabdoiden (Abb. 1c) bewaffnet und der Faden der $\mathrm{p}$-Rhabdoiden unbewaffnet ist (Abb. 8). Die $\mathrm{p}$-Rhabdoiden der Aktinien unterscheiden sich außerdem von den b-Rhabdoiden durch den deutlichen Querschnittsunterschied zwischen Schaft und Faden sowie durch die trichterförmige Offnung am distalen Ende des nicht entfalteten Schaftes (CARLGREN 1940).

\section{Die b-Rhabdoiden}

Die b-Rhabdoiden sind der häufigste Nesselkapseltyp der Aktinien. Sie können in allen Körperbereichen vorkommen und haben mit wenigen Ausnahmen einen ziemlich einheitlichen Bau. Die nicht entladene Kapsel hat einen geradlinig meist bis zum Boden der Kapsel reichenden schmalen Schaft (vgl. WeILl 1934, p. 53), an den sich ein dünnerer gewundener Faden anschließt. Der Faden füllt in engen Querwindungen die ganze Kapsel aus (Abb. 2d). Nach der Entladung werden an der dickwandigen Kapsel Klappen sichtbar (vgl. Westrall 1965). Der Schaft hat etwa die gleiche Länge wie die Kapsel und ist mit deutlichen Dornenwindungen und einem langen Faden versehen, 
der etwa die gleiche Breite wie der Schaft (Abb. 1c) und dessen 10-bis 20fache Länge aufweist.

Von diesem Bauplan weichen nur wenige b-Rhabdoiden ab: (1) In den Akontien der Aiptasiiden und in den Mesenterien verschiedener Aktiniiden kommen b-Rhabdoiden mit einer kürzeren, dichteren. Schaftbewaffnung und einem auffällig kurzen Faden vor (etwa 3- bis 6fache Schaftlänge). In geschlossenem Zustand tritt der Unterschied noch deutlicher hervor, da der Faden, kaum dünner als der Schaft, nur in wenigen Längswindungen einen Teil der Kapsel ausfüllt (Abb. 2c). (2) Die b-Rhabdoiden aus dem Scapus von Parantbus chromatoderus zeichnen sich ebenfalls durch einen kurzen kräftigen Faden aus. Der Schaft ist in entladenem Zustand und in nicht entladenem Zustand deutlich breiter als der Faden (Abb. 7a). (3) In den Akontien yon Metridium senile befinden sich die einzigen b-Rhabdoiden der Aktinien, deren Schaft nach der Entladung wesentlich länger ist als die Kapsel. Der Schaft besteht aus Falt- und Hauptstück. Bei der Entladung können Pfeile entstehen.

Im Gegensatz zu den p-Rhabdoiden sind Schaft und Faden der b-Rhabdoiden nach der Ausstülpung von einem schleimigen Sekret umhüllt (Abb. 1c). Dadurch werden unmittelbar nach der Entladung die Dornen verklebt und weisen mit ihrer Spitze zum Faden hin. Nach genügender Verdünnung des Sekretes stellen sie sich nach kurzer Zeit senkrecht zur Schaftoberfläche. Sie haben eine breite Basis und verjüngen sich im Gegensatz zu den Dornen der p-Rhabdoiden gleichmäßig zu einer scharfen Spitze.

\section{Die p-Rhabdoiden}

Der große Formenreichtum der p-Rhabdoiden erlaubt eine Einteilung in drei Kategorien. Diese Kategorien entsprechen verschiedenen Variationen, die WeILI bei den Mastigophoren und Amastigophoren beobachtet hatte (1934, Fig. 59-62).

Kategorie A: In unentladenem Zustand fällt dieser Rhabdoidentyp unter allen anderen Nematocysten durch den fehlenden Phasenkontrast seines flüssigen Kapselinhaltes auf (Abb. 7c). Dadurch hebt sich der stark kontrastierende Schaft sehr gut von seiner Umgebung ab. Der gerade Schaft reicht etwa bis in die Mitte der Kapsel und ist proximal zugespitzt. Seine grobe Schraubenstruktur tritt deutlich hervor.

Die Kapselwand ist so dünn, daß sie häufig nach der Entladung kollabiert (Abb. $7 \mathrm{~b}$ ). An der Kapselöffnung sind keine Klappen zu erkennen (Abb. 7b, 8). Der ausgestülpte Schaft hat etwa die Länge der Kapsel. Er ist selten kürzer oder länger, reicht jedoch nie über die doppelte Kapsellänge hinaus. Er hat kein Faltstück. Die Bewaffnung besteht aus deutlich abgesetzten Dornenwindungen. Die Dornen stehen senkrecht zur Schaftoberfläche. Ihre Länge entspricht etwa der jeweiligen Schaftbreite. Ohne Ubergang schließt sich ein dünner Faden an, der stets ausgestülpt wird.

Dieser Nesselkapseltyp wurde von WEILL als mikrobasische Mastigophore (1934, p. 562 ff.) und in einem einzigen Fall als mikrobasische Amastigophore (1934, Fig. 59; vgl. Werner 1965, Abb. 12) bei Sagartia elegans ( = S. miniata) beschrieben. Bei eigenen Untersuchungen an Sagartia elegans und zahlreichen anderen Aktinienarten habe ich jedoch den Faden nur in ausgestülptem Zustand angetroffen. Die Kategorie ist nur im Pharynx und in den Mesenterialfilamenten verschiedener Aktinienarten vertreten. 


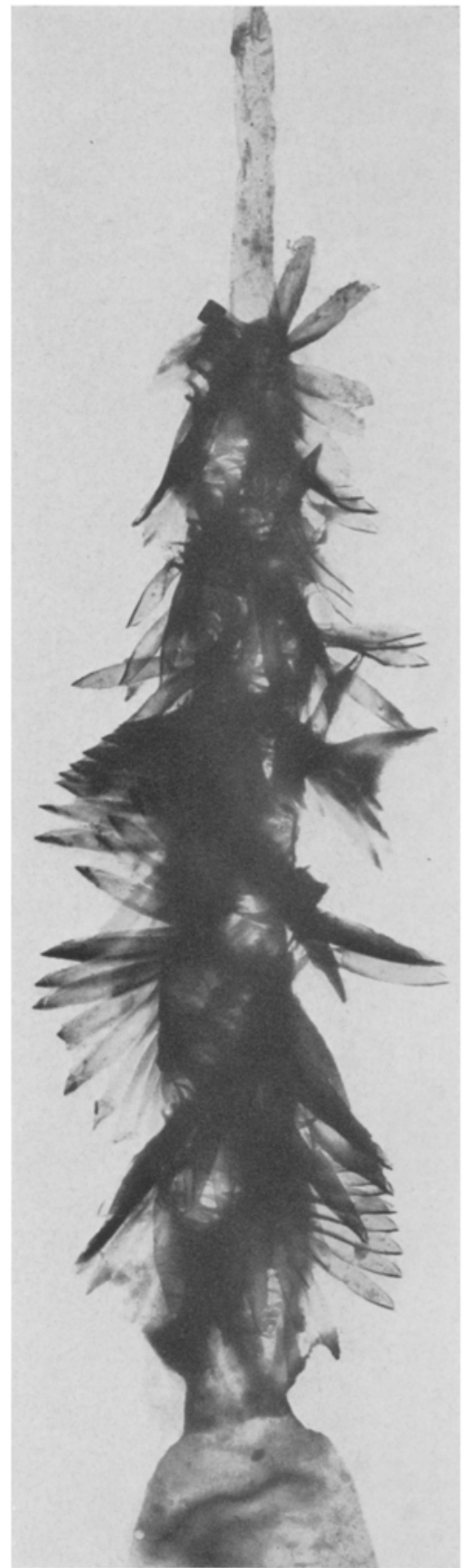

Abb. 8: p-Rhabdoide A, Tealia felma, Mesenterialfilament (5050:1) 
Kategorie B : Die unentladene Kapsel zeigt einen so starken Phasenkontrast, daß sich Schaft und Faden nur wenig von dem übrigen Kapselinhalt abheben ( $A b b .7 d$ ), Der Schaft reicht meistens bis in die Nähe des Kapselbodens, ist weitgehend isodiametrisch oder proximal zugespitzt und verfügt über eine feine Schraubenstruktur mit dichten Windungen (Abb. 3a, 4c). In einem Fall ist der Schaft länger als die Kapsel (Abb. 3a, Diadumene). Die entladene Kapsel hat eine dicke starre Wand und deutliche Klappen an der Offnung (Abb. 5, 7e-h, 9c). Der ausgestülpte Schaft variiert in seiner Länge zwischen einfacher und dreifacher Kapsellänge, kann jedoch auch bedeutend länger sein (Abb. 3b). Er besteht entweder nur aus dem Hauptstück oder aus Falt- und Hauptstück. Die dichte Bewaffnung setzt sich aus zahlreichen undeutlichen Dornenwindungen zusammen (Abb. 7e-g, 9a). Nach völliger Entladung wenden die Dornen ihre Spitzen gegen die Kapsel. Sie sind am Hauptstück länger als die jeweilige Schaftbreite. Der Schaft geht distal in ein konusförmiges unbewaffnetes Zwischenstück über, an welches sich häufig ein kräftiger Faden sehr unterschiedlicher Länge anschließt. In einigen Fällen bleibt der Faden nach der Entladung in der Kapsel zurück. Bei einem Teil der Nesselkapseln dieser Kategorie Können bei der Evagination Pfeile entstehen.

Diese Kategorie katn in allen Bereichen des Aktinienkörpers vorkommen. Sie kann nach der Länge und Gestalt des Schaftes und der Lage des Fadens nach der Entladung weiter aufgegliedert werden.

Folgende Kombinationen kommen vor:

(1) Schaft etwa kapsellang, ohne Faltstïck;

(a) mit stets ausgestïlptem langen Faden (Abb. 7e);

(b) Faden stets in der Kapsel verbleibend (Abb. 7f).

(2) Schaft kapsellang, häufig länger, mit Faltstück;

(a) Faltstück kürzer als Hauptstück oder gleichlang, Faden ausgestülpt oder in der Kapsel gerade gestreckt, kürzer als die Kapsel (Abb. 7d, 5), Pfeilbildung möglich;

(b) Faltstück länger als Hauptstück, Faden ausgestülpt, keine Pfeilbildung, Schaft in nicht entladenem Zustand länger als die Kapsel, Faltstück in der vorderen Kapselhälfte aufgerollt (Abb. $3 a, b$ ).

Die Nesselkapseln der Kategorie B wurden mit der Ausnahme der Unterkategorie $2 \mathrm{~b}$ bisher als mikrobasische Mastigophoren bzw. Amastigophoren bezeichnet (WEILL 1934, Carlgren 1940, 1945 u. a.). Die Variante $2 b$ führte Weill (1934, p. 582) zunächst als „Rhabdoides indéterminées“. Sie wurde später von CARLGREN (1940) den makrobasischen Amastigophoren zugeordnet, obwohl CARLgren die Kapsel nicht in entladenem Zustand beobachtet hat.

Kategorie C: Die geschlossene Kapsel hebt sich durch Phasenkontrast von der Umgebung ab. Der Schaft ist häufig länger als die Kapsel, daher gebogen oder in Längswindungen aufgerollt (Abb. 10b) und deutlich anisodiametrisch (Abb. $3 c$ ). Mit seinem distalwärts abnehmenden Querschnitt verringert sich auch der proximal stark aufgelockerte Abstand der Schraubenwindungen (Abb. 11).

Die entladene dickwandige Kapsel hat deutliche Offnungsklappen. Der ausgestülpte Schaft ist stets länger als die Kapsel. Haupt- und Faltstück unterscheiden sich entweder nur unwesentlich durch stärkere Faltung am Faltstiick oder deutlich durch fehlende Bewaffnung am Faltstück (Abb. 10c, 11). Auf ein kleines konusförmiges 

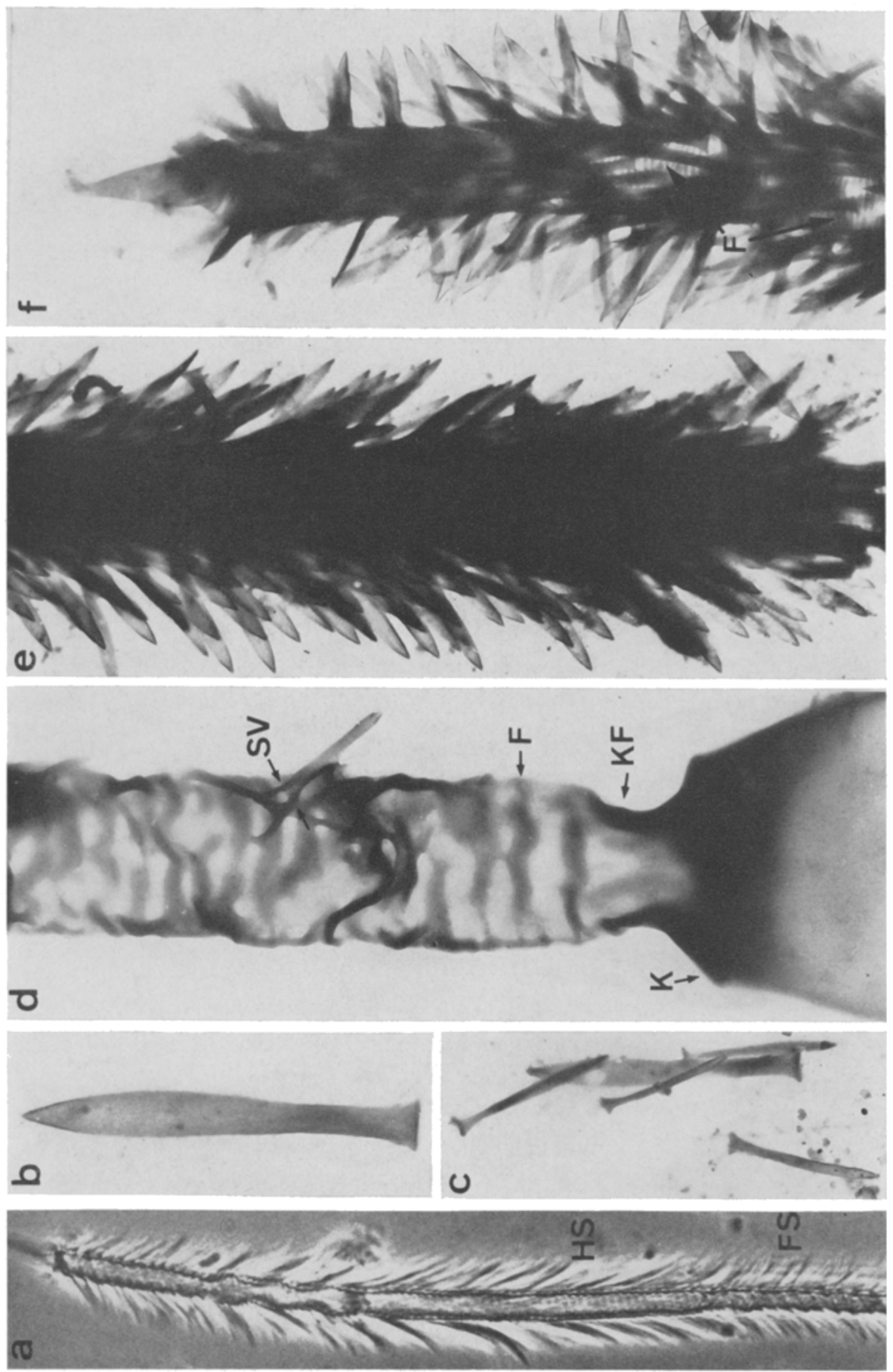
Zwischenstück folgt entweder ein kurzer kräftiger Faden oder der Faden bleibt nach der Evagination in der Kapsel liegen. Die Bewaffnung besteht aus deutlich abgesetzten ziemlich einheitlichen Dornwindungen (Abb. 3d, 10d). Die Dornen sind etwas kürzer als der jeweilige Schaftquerschnitt und stehen senkrecht zur Schaftoberfläche. Während der Entladung entstehen keine Pfeile.

Die Nesselkapseln der Kategorie C sind nur aus den Tentakeln und dem Scapus einzelner Aktinienarten bekannt und noch wenig erforscht. Von einer weiteren Unterteilung soll vorläufig abgesehen werden, da die genaue Verbreitung noch zu wenig bekannt ist. WeILL schuf für diesen Nesselkapseltypus die Kategorie der makrobasischen Amastigophoren. Einige mikrobasische Amastigophoren CARLGRENs (1940) weisen jedoch ebenfalls die typischen Kennzeichen dieser Kategorie auf und stülpen außerdem nach der Entladung einen kurzen Faden aus (Abb. 3d). Die Darstellung des Faltstückes auf WeILLs Abbildung 62b (1934, p. 69; vgl. WeRnER 1965, Abb. 13a) eñtspricht nicht den wirklichen Verhältnissen. Das Faltstück dieser Kategorie ist immer breiter als das Hauptstück (Abb. 11). Curress (1955) betrachtet die Kategorie als ein Relikt innerhalb der Actiniaria. Die Nesselkapsel hat insofern einen ursprünglichen Charakter, als sich der Schaf, insbesondere aber das Faltstück, während der Reifung weniger im Verhältnis zur Kapsel verkürzt als bei anderen Kategorien (Abb. 10a, b). Die Verlängerung des Schaftes ist daher nach der Evagination entsprechend geringer als bei vergleichbaren Nesselkapseln der Kategorie B. In den meisten Kapseln der Kategorie $C$ ist der Schaft in vierfachen Längswindungen aufgerollt, so daß das distale Schaftende in der Nähe der Kapselöffnung liegt (Abb. 10b). Im Verhältnis zur Kapsel ist jedoch die Länge des Schaftes in entladenem Zustand etwa die gleiche oder sogar geringer als bei der Variante $2 b$ der Kategorie $B$, deren Schaft stets nur in eineinhalbfachen Längswindungen beobachtet wurde (Abb. 3a, vgl. WeILl 1934, Fig. 393; CarLGREN 1940, Fig. XI; HAND 1955, Fig. 36). Die Nesselkapseln von Diadumene cincta haben z. B. nach der Entladung einen Schaft von 7- bis 8 facher Kapsellänge, die Nesselkapseln von Alicia hingegen nur 4- bis 6fache Kapsellänge. WeilL 1934, p. 70 maß bei Leburniat 4- bis 8fache Kapsellänge.

Das unterschiedliche Ausmaß der Schaftverlängerung spiegelt sich bei beiden $\mathrm{Ka}$ tegorien auch an dem Schaft der geschlossenen Kapsel wieder. Die Schraubenstruktur ist bei der Kategorie B dichter als bei der Kategorie C (Abb. 3a, c; 4c). Bei der Kategorie $B$ ist im Gegensatz zu der Kategorie C das Faltstück stets mit dichteren Schraubenwindungen versehen als das Hauptstück (Abb. 5). Die lappigen Schraubenwindungen des Faltstïckes der Kategorie $C$ sind am Rand mit blasenartigen Ausstïlpungen versehen (Abb. 3c, 11). Außerdem ist die Schaftwandung noch einmal in kleine Falten gelegt, deren Abstand sich ebenfalls gegen das distale Schaftende verkürzt. Der Schaft enthält das gleiche elektronendichte Matrixmaterial wie die Kapsel.

Abb. 9: (a) p-Rhabdoide B 2a, Aiptasia diaphana, Akontium: Hauptstiick (HS) mit dichten Dornenwindungen, Faltstück (FS) mit weniger dichten Dornenwindungen (1750:1); (b) $\mathrm{p}^{-}$ Rhabdoide B $2 a$, Metridium senile, Akontium: Dorn des Hauptstückes $(14450: 1) ;$ (c) $\mathrm{p}^{-}$ Rhabdoide B 2a, M. senile, Dornen des Faltstidkes, im Hintergrund ein Dorn des Hauptstïckes $(10100: 1)$; (d) p-Rhabdoide B 2a, M. senile, Faltstück durch "Pfeilbildung" entwaffnet, Falten ( $\mathrm{F}$ ), einzelner Dorn mit scharnierfömiger Verankerung (SV), Klappen $(\mathrm{K})$ mit Fortsätzen (KF) $(12400: 1)$; (e) p-Rhabdoide B 2a, M. senile, Hauptstuck $(6300: 1)$; (f) p-Rhabdoide, distales Schaftende, Falten (F) mit Dornenansatz (vgl. Abb. 9d) $(6650: 1$ ) 

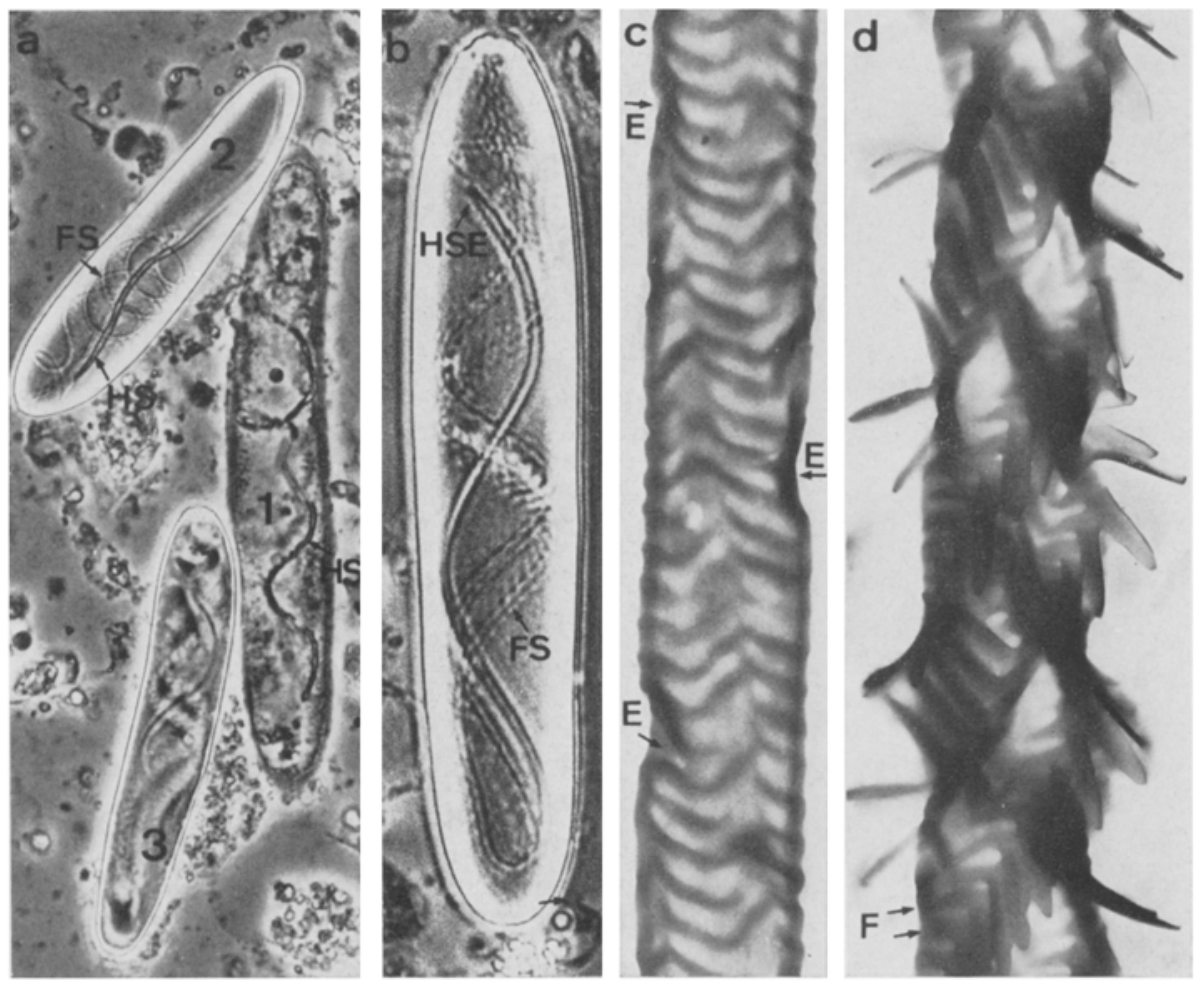

Abb. 10: (a) p-Rhabdoide C, Alicia costae, Scapus: drei verschiedene Reifestadien (585:1); Stadium 1: Kapsel und Schlauch vor der Kontraktion (rgl. Abb. 4a, b), nur Hauptstuick (HS) sichtbar; Stadium 2: Kapsel in der Länge verschrumpat, transparent, Haupt- (HS) und Faltstiick (FS) deutlich zu erkennen; Stadium 3: Kapsel fast reif, in der Breite geschrumpt, weniger transparent, Schlauch daher weniger deutlich sichtbar; (b) p-Rhabdoide C, A. costae, reife Nesselkapsel, Faltstück (FS), distales Ende des Hauptstïckes (HSE), Phasenkontrast abgeblendet (1150:1); (c) p-Rhabdoide C, A. costae, Faltstück mit typishen Eindellungen (E) $8560: 1)$; (d) p-Rhabdoide C, A. costae, Hauptstück, Falten (F) mit inserierenden Dornen $(10700: 1)$

In entladenem Zustand entsprechen die Dornenwindungen den Schraubenwindungen und die in bestimmten Abständen wiederkehrenden Eindellungen am Faltstück den blasenartigen Ausstullpungen (Abb. 10c, d). Die feine Faltung des Schaftes ist ebenfalls in entladenem Zustand zu erkennen. Der Faltenabstand ist am Hauptstück dichter als am Faltstiick. Die Falten verlaufen nicht genau horizontal, sondern gewellt (Abb. 10c). In ihnen inserieren die Dornen (Abb. 10d).

Bei der Kategorie B konnte auch Westrall (1966, Fig. 12, 13) eine feine Faltung der Schaftwand der geschlossenen Kapsel feststellen. Am entladenen Schaft sind die Falten im Bereich des Faltstückes gröber und weniger dicht als an dem besonders dicht bedornten Hauptstück (Abb. 9d-f).

An dem ausgestülpten Schaft der Kategorie A konnte ich dagegen nicht feststellen, daß die Dornen in derartigen Falten inserieren (Abb. 8). Alle drei Kategorien besitzen ziemlich ähnlich gebaute Dornen, die wesentlich von denjenigen der b-Rhab- 


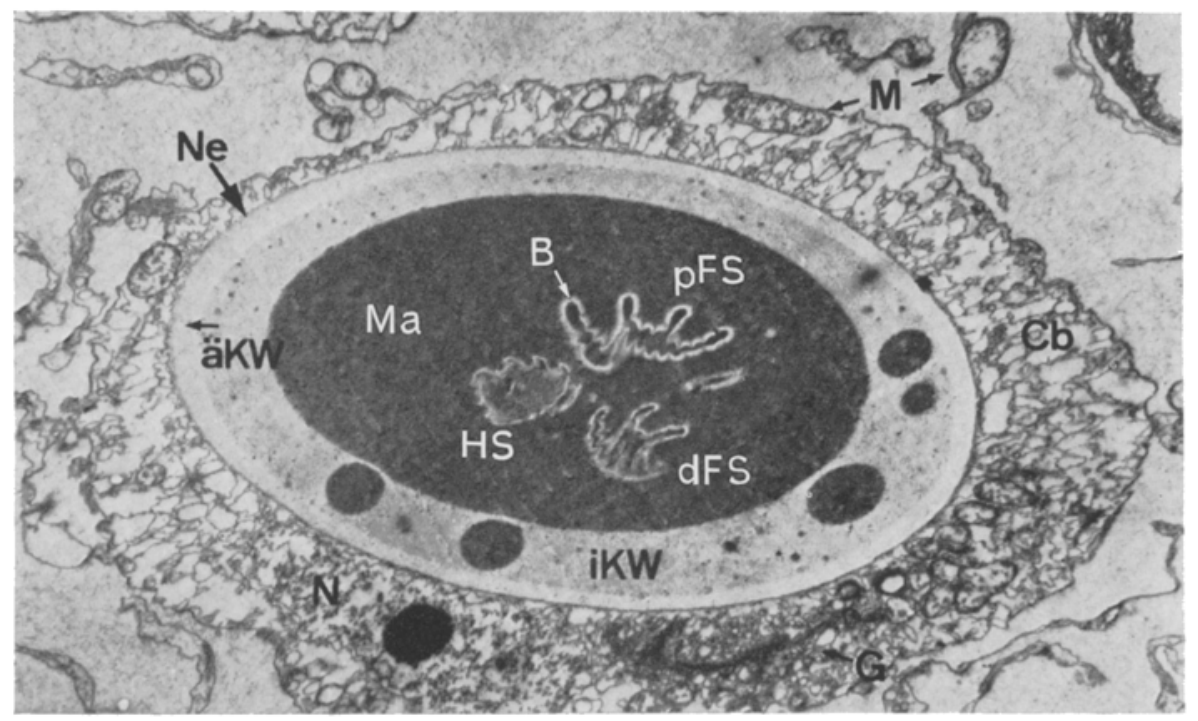

Abb. 11: p-Rhabdoide C, Alicia costae, Scapus: Schrägschnitt durd den unteren Teil der Nesselkapsel (Ne), Schaft besteht nur aus dreieinhalbfachen Längswindungen, daher im unteren Teil nur drei Querschnitte, äußere Kapselwand (äKW), innere Kapselwand (iKW) mit Matrixeinschlüssen, Matrix (Ma), proximaler Teil des Faltstückes (pFS), blasenartige Ausstülpungen (B) an den weiten Schraubenwindungen, distaler Teil des Faltstüdkes (dFS) mit engeren Schraubenwindungen, Hauptstick (HS) Dornen enthaltend, Schraubenwindungen wenig ausgeprägt, Cnidoblast (Cb), Kern (N), Golgiapparat (G), Mitochondrien (M) (2730:1)

doiden abweichen. Die lanzettförmige flache Spitze ist durch einen dickeren Stil mit einer mehr oder weniger breiten Basis verbunden ( $\mathrm{Abb}, 9 \mathrm{~b}$ ).

\section{Die diagnostische Bedeutung für die Systematik}

Der diagnostische Wert der einzelnen Nesselkapseltypen ist besser durch Vergleich mit den morphologischen und ökologischen Gegebenheiten der verschiedenen Aktinienarten darzustellen, was z. Z. in einer ausführlicheren Arbeit behandelt wird. Hier sollen nur allgemeine Gesetzmäßigkeiten und einige typische Beispicle aufgezeigt werden, um den Sinn der vorliegenden Neugruppierung vor Augen zu führen.

Der taxonomische Wert der Spirocysten ist äußerst gering. Nach meinen Untersuchungen weisen die Spirocysten die meisten individuellen Größenunterschiede auf und kommen in ziemlich einheitlicher Form bei fast allen Aktinienarten vor.

Bisher ist die Formenvielfalt der Haplonemen für systematische Zwecke kaum berücksichtigt worden, da ihre Verbreitung zu wenig bekannt ist. HAND (1966) ist der Meinung, daß Haplonemen innerhalb der Actiniaria selten vorkämen und nur auf die Fangtentakeln einiger Acontiaria und auf die Akrorhagen der Actiniidae beschränkt seien. Im Scapus verschiedener Acontiaria wurden die Haplonemen bisher wegen ihres kontrastreichen Inhalts, ihrer schwierig zu erzielenden Entladung und ihrer meist gerin- 
gen Größe übersehen oder nicht eindeutig erkannt (HAND 1961a). Bei der Beschreibung z. B. des Cnidoms von Sagartia troglodytes, Cereus pedunculatus, Metriditum senile, Diadumene cincta und verschiedenen Aiptasiiden (Abb. 1d) blieben sie unberücksichtigt (Weill 1934, Carlgren 1940, 1945, Hand 1955).

Typische isorhize Haplonemen sind das Charakteristikum echter Akrorhagen vieler Actiniidae, so unter anderem der Gattung Anthopleura (Abb. 2a). Einige Arten dieser Gattung weisen zusätzlich anisorhize Haplonemen im Scapus auf. Bei Anthopleura (Bunodactis) rubripunctata und deren Jugendform Bunodactis sabelloides kommen jedoch sehr typische Anisorhizen in den Akrorhagen vor (Abb. 2b, vgl. ScHmidT 1967).

Große dünnwandige Haplonemen (Abb. 1e-g) haben eine eigenartige Verbreitung. Sie kommen in wechselnder Häufigkeit in fast gleicher Größe in so verschiedenen Aktinien vor, wie Anemonia sulcata (Scapus), Sagartiogeton undatus (Scapus), Antbopleura rubripunctata (Akrorhagen), Sagartia troglodytes (Akontien) (vgl. WEILL 1934, Stephenson 1929). Das sporadische Auftreten in den Fangtentakeln ist nur von bedingter taxonomischer Bedeutung.

Die b-Rhabdoiden variieren nur mit wenigen Ausnahmen, die allerdings spezifischen systematischen Wert haben. Sie zeigen meist eine typische Form in den einzelnen Organen der verschiedenen Aktinienfamilien. So kennzeichnet eine sehr charakteristische kegelförmige b-Rhabdoide die Akontien der Gattung Telmatactis (vgl. CuTress 1955, Fig. 4g).

Die p-Rhabdoiden haben auf Grund ihrer typischen Verbreitung und ihrer differenzierten Form unter allen Nesselkapseln die größte Bedeutung für die Taxonomie. Der Wert der neuen Kategorien und Varianten wird am besten anhand der Tabellen 2 und 3 demonstriert.

Nach Tabelle 2 sind die Boloceroididae, Isophelliidae, Sagartiidae, Metridiidae und Aiptasiidae ebensowenig zu unterscheiden wie die Andresiidae, Actiniidae, Actinistolidae und Hormathiidae einerseits und die Aliciidae und Diadumenidae andererseits. In der Tabelle 3 stimmen die Boloceroididae mit den Aliciidae, die meisten Actiniidae mit den Andresiidae und die Actinostolidae und Sagartiidae mit den Metridiidae überein. Die letzten drei Familien unterscheiden sich aber durch spezielle b-Rhabdoiden (Actinostolidae, Metrididae) oder durch das Auftreten von Haplonemen im Scapus (Sagartiidae, Metridiidae) voneinander. WeIL (1934, p. 591) war der Meinung, daß die Metridiidae und Aiptasiidae besser übereinstimmten als eine dieser Familien mit den Sagartiidae. Der Tabelle 3 ist aber eher das Gegenteil zu entnehmen, da den Aiptasiiden die Kategorie A fehlt. WeILL (1934, p. 594) erkannte dagegen die große Ahnlichkeit des Cnidoms von Diadumene cincta mit demjenigen der Aiptasiden. Die Andresiiden und die meisten Actiniiden besitzen gleiche Nesselkapseltypen. Unterschiede bestehen nur in der Form der b-Rhabdoiden. Form- und typenmäßig stimmen dagegen die Nesselkapseln mancher Boloceroididae, z. B. von Bunodeopsis, wesentlich besser mit denjenigen von Alicia überein, als mit denjenigen von Boloceroides, obwohl auch diese Gattung im Gegensatz zu den Angaben Carlgrens (1940) über die Variante $1 \mathrm{a}$ in den Mesenterialfilamenten verfügt. Die nahe Verwandtschaft von Alicia und Bunodeopsis war schon von STEPHENSON (1921) und CARLGREN (1940) vermutet worden. 
Tabelle 2

Die Verteilung Werlls Kategorien nach CarLgReN (1949)

\begin{tabular}{|c|c|c|c|}
\hline Familie & $\begin{array}{c}\text { Mikrobasische } \\
\text { p-Mastigophoren }\end{array}$ & $\begin{array}{l}\text { Mikrobasische } \\
\text { Amastigophoren }\end{array}$ & $\begin{array}{l}\text { Makrobasische } \\
\text { Amastigophoren }\end{array}$ \\
\hline Andresiidae & + & & \\
\hline Boloceroididae & + & + & \\
\hline Aliciidae & + & + & + \\
\hline Actiniidae & + & & \\
\hline Actinostolidae & + & & \\
\hline Isophelliidae & + & + & \\
\hline Hormathiidae & + & & \\
\hline Sagartiidae & + & + & \\
\hline Metridiidae & + & + & \\
\hline Aiptasiidae & + & + & \\
\hline Diadumenidae & + & + & + \\
\hline
\end{tabular}

Tabelle 3

Die Verteilung der neuen Kategorien und Varianten

\begin{tabular}{|lccc|}
\hline Familie & Kategorie A & Kategorie B & Kategorie C \\
\hline Andresiidae & + & $+1 \mathrm{a}, 2 \mathrm{a}$ & + \\
Boloceroididae & & $+1 \mathrm{a}, 2 \mathrm{a}$ & + \\
Aliciidae & + & $(+1 \mathrm{a})^{: 5}$ & \\
Actiniidae & + & $+1 \mathrm{a}, 2 \mathrm{a}$ & \\
Actinostolidae & + & $+1 \mathrm{a}, 1 \mathrm{~b}$ & \\
Isophelliidae & + & $+1 \mathrm{a}, 2 \mathrm{a}$ & \\
Hormathiidae & + & $+1 \mathrm{a}, 2 \mathrm{a}$ & \\
Sagartiidae & $+1 \mathrm{a}, 2 \mathrm{a}$ & \\
Metridiidae & & $+1 \mathrm{a}, 2 \mathrm{a}, 2 \mathrm{~b}$ & \\
Aiptasidae & & & \\
Diadumenidae & & & \\
$*$ Bisher nur aus Actinia equina & und Anthopleura rubripunctata bekannt. \\
\hline
\end{tabular}

Die Variante 1a der Kategorie B kommt in ovoider oder ellipsoider Form in den Mesenterialfilamenten der meisten Aktinien vor, ausgesprochen selten jedoch in der Familie der Acrinidae. Als einziger Vertreter der Kategorie B kommt z. B. die Variante $1 \mathrm{a}$ in den Mesenterialfilamenten von Actinia equina aus dem Mittelmeer (Banyuls, Dubrovnik, Neapel, Palermo, Stromboli), und zwar sowohl in der viviparen als auch in der oviparen Form vor (vgl. Schmid 1967a). Sie fehlt dagegen in Actinia equina aus der Nordsee (Helgoland) sowie in Actinia cari und Actinia equina var. striata aus dem Mittelmeer (Banyuls, Dubrovnik). Actinia cari konnte bisher nicht eindeutig von Actinia equina unterschieden werden. Im Gegensatz zu Actinia equina kommen Actinia cari und Actinia equina var. striata nur im Infralitoral vor, Actinia equina var. striata sogar meist in Tiefen von 8-12 m.

Die heterogene Gattung Sagartiogeton (Sagartiidae) umfaßt teilweise Arten, die einwandfrei zur Familie der Aiptasiidae gehören, wie z. B. Aiptasiogeton (Sagartio- 
geton) n. g. laceratus. Die Art war schon vor ANDres (1884) mit Recht, wie heute anhand des Cnidoms beweisbar, als Aiptasie bezeichnet worden. Aiptasiogeton besitzt die gleichen Haplonemen im Scapus (vgl. Abb. 1d-g) und die gleichen b-Rhabdoiden in den Akontien (vgl. Abb. 2c, d) wie die Aiptasien. Ebenso fehlt dieser Aktinie die Kategorie $A$, und in Obereinstimmung mit den Aiptasien neigen die p-Rhabdoiden der Kategorie B (2a) aus den Akontien sehr stark zur Pfeilbildung.

Die Länge des ausgestülpten Schaftes ist häufig art- oder gattungsspezifisch. Bei den Aiptasien, Cerews pedunculatus und Sagartia elegans ist der Schaft der Kategorie B (2a) aus den Akontien etwa ein Viertel bis ein Drittel länger als die Kapsel, bei Aiptasiogeton laceratus dagegen doppelt so lang und bei Sagartia troglodytes etwa gleichlang wie die Kapsel. Artspezifisch ist auch die Verteilung der Kategorie $B$ in den Tentakeln der Diadumenidae. Nach HAND (1955) kommt die Variante $2 \mathrm{~b}$ außer bei Diadumene cincta auch bei Diadumene lighti vor. Diadumene franciscana und D. leucolena verfügen dagegen nur über die Variante 2a. Ahnliche Unterschiede weisen die Vertreter der Kategorie C bei der Gattung Bunodeopsis auf. Bei Bunodeopsis globulifera ist der Schaft in der Kapsel im Gegensatz zu B. strumosa drei- bis vierfach in der Kapsel aufgerollt und in entladenem Zustand entsprechend länger.

\section{DISKUSSION}

\section{Der Entladungsmechanismus}

Der Vorgang der Entladung kann sehr gut an den p-Rhabdoiden der Kategorie B, die in nicht entladenem Zustand deutlichen Phasenkontrast aufweisen (Abb. 7d), verfolgt werden, wenn die Entleerung des flüssigen Kapselinhaltes nach der Entladung verhindert, der Schlauch aber ausgestiilpt ist. In diesem Zustand ist der Phasenkontrast etwas verringert, da sich der Kapselinhalt verdünnt und auf das Volumen von Schlauch und Kapsel verteilt hat. Der Innendruck ist jedoch immer noch wesentlich höher als der Außendruck, wie man an der maximal gedehnten Kapsel, dem maximal gestreckten Schlauch und den senkrecht zur Schaftoberfläche stehenden Dornen erkennen kann. Erst nach der Entleerung des Kapselinhaltes am Schlauchende schrumpt nicht nur die Kapsel, sondern verkürzt sich auch der Schlauch. Gleichzeitig nehmen die Dornen die für die Kategorie B typische, zur Kapsel gerichtete Stellung ein. Der Vorgang ist sehr ähnlich auch bei den anderen Nesselkapseltypen zu beobachten.

Der Befund beweist, daß nicht die Ausdehnung der Schaftwandung (Westrall 1965), sondern die Erhöhung des osmotischen Druckes in der Kapsel die Hauptursache der Schlauchausstülpung darstellt. Gleiches gilt auch für isolierte getrocknete Schlauchteile, an denen RoBson (1953) demonstrierte, daß sie sich in nicht umgestulptem $\mathrm{Zu}$ stand unter Wasseraufnahme verlängern. Der Grund liegt in der Tatsache, daß der Schlauch ebenfalls über einen quellfähigen Inhalt verfügt. Ich teile daher die Ansicht RoBsons, daß ein hypotonisches Medium auch an der Offnung des sich entladenden Schlauches wirkt. Es ist allerdings sehr zweifelhaft, ob Quelleisten den Schlauch auszustülpen vermögen, indem sie an der Schlauchöffnung stark aufquellen (WILL 1909, 
1914, vgl. auch MERGNer 1964). Die Spirocysten der Aktinien schleudern jedenfalls in den meisten Fällen den Schlauch explosionsartig in einem Bogen aus, wodurch der Schlauchinhalt netzartig ausgebreitet wird (vgl. WiLL 1909, Taf. III, Abb. 4). Daher sind Bilder wie die Abbildungen $1 \mathrm{a}$ und $\mathrm{b}$ nur selten zu beobachten.

Ich bin mit Picken \& Skazr (1966) der Meinung, daß auch im lebenden Gewebe die Erhöhung des Innendruckes die Entladung der Nesselkapseln verursacht, da ich meine Beobachtungen gelegentlich auch an Nesselkapseln in lebenden Filamenten oder Akontien in unverdünntem Seewasser machen konnte. Die unterschiedliche Stellung der Dornen zeigt an, daß nicht nur die Schraubenwindungen, sondern auch die Falten, in welchen die Dornen inserieren, verstrichen sind, solange der Druck anhält. Die Falten treten jedoch nach dem. Druckausgleich wieder hervor, wie die Ausrichtung der Dornen zur Kapsel hin beweist. Bei den p-Rhabdoiden der Kategorie B von Diadumene cincta und bei den speziellen b-Rhabdoiden von Paranthus cbromatoderus legt sich nach der vollständigen Kapselentleerung auch der Faden in deutliche Falten (Abb. 7a). Bei den anderen Kategorien (A und C) der $\mathrm{p}$-Rhabdoiden stehen die Dornen senkrecht auf dem Schaft. Der Schaft der Kategorie A besitzt auch nach völliger Entleerung und Trocknung keine Falten. Aus methodischen Gründen konnte leider noch nicht untersucht werden, ob die großen Falten am Schaft der Kategorie $\mathrm{C}$ im wäßrigen Medium verstreichen.

Ob der Binnendruck, der den Schaft der Rhabdoiden explosionsartig ausschleudert und bei der geringsten Hemmung sogar deformiert, während der Entladung nachläßt, ist fraglich (vgl. Prcken 1953). Der kurze Faden der p-Rhabdoiden entlädt sich gleichmäßig und praktisch mit der gleichen Geschwindigkeit wie der Schaft. Bei den b-Rhabdoiden vollzieht sich die Ausstuilpung des meist sehr langen Fadens wesentlich langsamer als die des Schaftes. Der Faden wird ruckartig unter schraubenförmigen Rechtsdrehungen ausgestülpt (vgl. RoBson 1953). Da er bis zur vollständigen Entladung gegenüber dem umgebenden Medium Phasenkontrast beibehält, muß auch hier als treibende Kraft in erster Linie der quellende Inhalt betrachtet werden. Die Verlangsamung der Bewegung hat ihre Ursache eher in der großen Fadenlänge als in einem Nachlassen des Innendruckes. Der Druck hält auch bei den b-Rhabdoiden die Kapsel und den gesamten Schlauch bis zur völligen Ausstülpung unter Spannung. Anderenfalls würden Schlauch und Kapsel vorher kollabieren und somit die Entlassung einer größeren Menge Nesselgiftes am Schlauchende unmöglich machen. Die Elastizität von Kapsel und Schlauch wirkt sich also erst bei der Entleerung des flüssigen Kapselinhaltes aus und nicht bei der Schlauchausstülpung.

Der Entladungsmechanismus kann nur an unbeschädigten Nesselkapseln funktionieren, da ein frühzeitiges Ausfließen des Kapselinhaltes die Ausstülpung verhindert bzw. unterbricht. Es ist daher nicht möglich, nach Eröffnung der Kapselwand die Nesselkapsel noch zur Entladung zu bringen. Gegen diesen Befund sprechen die Beobachtungen von WILL (1909) und MergNer (1964), daß das Kapselsekret schon während der Entladung durch die Schlauchwand austritt. Die elektronenmikroskopischen Bilder (Abb. 7, 8d, f, 9c, d) lassen keinerlei Poren (WILl 1909) erkennen. Andererseits ist aber der Schlauch der b-Rhabdoiden nach der Entladung von einer schleimartigen Substanz umhüllt (Abb. 1c) und der Schaft in der Kapsel mit dem gleichen elektronendichten Material gefüllt wie die Kapsel (Abb. 10). Es handelt sich demnach bei den 
Befunden von WILL (1909) und MERGNER (1964) nicht um ausgetretenen Kapselinhalt, sondern um Schaftinhalt.

Ebenso funktioniert der Entladungsmechanismus nur an reifen Nesselkapseln, da diese ihren Inhalt genügend verdichtet und ihre Wandung genügend erhärtet haben, wie der unterschiedliche Phasenkontrast und die Volumenabnahme mit fortschreitender Reifung zeigen (Abb. 9a). Die erstmals von. IwANzoff (1896) beobachtete Volumenverringerung war von WEILL (1934, p. 269) in Zweifel gezogen worden, da sie bisher an reifenden Nesselkapseln der Hydrozoen nicht beobachtet worden ist (SCHNEIDER 1900, Mergner 1964, Günzl 1968). Im Gegensatz zu Schneider beobachteten MERGNER und GüNZL sogar eine Vergrößerung der Kapsel mit zunehmender Reife.

GüNZL (1968) demonstrierte die Elastizität der Kapselwand von Stenotelen aus Dipurena reesi, indem er mit verdünnter Essigsäure (Verdünnungsgrad nicht angegeben) das Nesselgift ( = flüssiger Kapselinhalt) ausfällte und danach eine Verkleinerung der Kapsel konstatierte. Nach meinen Beobachtungen ist ein solcher Effekt an nicht entladenen Nesselkapseln der Aktinien mit etwa halbverdünnter Essigsäure zu erzielen. Leicht zu entladende Nesselkapseln explodieren dabei vor der Fällung des Kapselinhaltes noch etwa bis zur Hälfte des Schaftes, andere schwellen kurz an und schrumpfen anschließend. Derartige behandelte Nesselkapseln entladen sich ebensowenig wie beschädigte, da die Fällung irreversibel ist (GünzL 1968).

\section{Die Neueinteilung}

Der unterschiedliche Querschnitt der nicht entladenen Schlauchabschnitte und die deutlich abgesetzte Bewaffnung des ausgestülpten Schlauches gestatten bei den Heteronemen eine Unterteilung des Schlauches in Schaft und Faden. Beide Merkmale trennen im Gegensatz zu der bisherigen Definition eindeutig Haplonemen und Heteronemen. Der Faden sollte daher nicht als Schlauch bezeichnet werden (WERNER 1965), da er nur einen Teil desselben darstellt, und dieser Terminus auch für den gesamten Nesselapparat der Haplonemen gilt.

Die lange Bedornung bedingt den geradlinigen Verlauf des Schaftes oder zumindest seines Hauptstückes (Hand 1961). Nach der Definition von Cutress (1955) sollen aber gerade die „Basitrichen“ einen vollständig aufgerollten Schlauch haben, obwohl WeILL (1934, p. 53) ausdrücklich hervorhebt, daß die basitrichen Haplonemen und alle Heteronemen mit Ausnahme der „makrobasischen Kategorien“ über einen gerade gestreckten Schaft (= Achsenkörper) verfügen. Es überrascht daher, daß HAND (1961) die von Cutress (1955, Fig. 3) abgebildeten Beispiele als Basitriche "in every sense" betrachtet, obwohl er als erster den Zusammenhang zwischen der abgesetzten langen Bewaffnung und dem in der Kapsel geradlinig verlaufenden Schaft richtig erkannte. Bei der Kategoric B 2b und den meisten Nesselkapseln der Kategorie C - beide ehemalige makrobasische Amastigophoren - bedingt die besondere Schaftlänge ein Aufrollen des Faltstückes in Längswindungen.

Die vorliegende Neugruppierung eliminiert alle Kategorien, die WerLL für die isorhizen und anisorhizen Haplonemen errichtet hatte. Im Gegensatz zu Werner. (1965) vertrete idh die Auffassung, daß die ehemaligen Atrichen und Holotrichen nicht 
mehr als getrennte Kategorien zu betrachten sind, da der Beweis für die Existenz völlig unbewaffneter Nematocysten bislang aussteht.

Bei den Haplonemen stehen in entladenem Zustand Schlauchbreite und Dornengröße in direktem Verhältnis zueinander (SKAER \& PICKEN 1965). Entsprechende Kaliberunterschiede sind auch am Schlauch der unentladenen Kapsel zu erkennen. Es ist daher unwahrscheinlich, daß es Haplonemen mit einheitlicher Dornenlänge aber unterschiedlicher Schlauchbreite gibt (homotriche Anisorhize, Werll 1934). Derartige Haplonemen kommen bei den Aktinien nicht vor. Schon eine geringgradige Vergrößerung der Dornen bedingt eine Verbreiterung des Schlauches, wie ich besonders an den anisorhizen Haplonemen aus den Akrorhagen von Anthopleura rubripunctata beobachten konnte (Abb. 2b). Daher sind anisorhize Haplonemen im Lichtmikroskop ohne weiteres von den weitgehend isorhizen Haplonemen zu unterscheiden (Abb. 2a).

Mit der Einteilung der rhabdoiden Heteronemen in $b$ - und $p$-Rhabdoiden werden Nesselkapseln unterschieden, die schon STEPHENson (1929) als gesonderte Typen, sogenannte "Spirulae" bzw. „Penicilli", bei den Aktinien erkannt hatte. Wie die Befunde bestätigen, hat STEPHENSON mit Recht der An- oder Abwesenheit eines Fadens bei entladenen p-Rhabdoiden (Penicilli) keine besondere Bedeutung beigemessen. WeIlls harte Kritik an STEPHENson besteht nicht zuletzt deshalb zu Unrecht, als sich auch Weill (1934, p. 68) keineswegs darüber Klarheit verschaffen konnte, ob der Schaft in der geschlossenen Kapsel einen Faden hat oder nicht. Heute ist es möglich, den Faden auch innerhalb der Kapsel bei allen p-Rhabdoiden nachzuweisen ( $\mathrm{Abb} .4 \mathrm{c}, 7 \mathrm{~g}$ ). Somit ist auch eine Verwechslung mit den Haplonemen ausgeschlossen (vgl. WeIL 1934, p. 71; Carlgren 1940, p. 53; Cutress 1955, p. 133, 134; Skaer \& Picken 1965, p. 139). Ich bin wie Cutress der Auffassung, daß kein Unterschied zwischen Amastigophoren und Mastigophoren besteht, und daher beide als Kategorien eliminiert werden sollten, denn alle Heteronemen sind Mastigophoren. Hand (1961) hingegen sprach sich für die Beibehaltung der Amastigophoren aus, da sie sich nach seiner Ansicht durch einen besonders kurzen Faden genügend von den Mastigophoren unterscheiden. Die Länge des Fadens eignet sich aber ebensowenig wie die des Schaftes zur Einteilung der Rhabdoiden.

\section{Die Bedeutung für die Systematik und Phylogenie}

Von allen Nesselkapseln sind die drei Kategorien der p-Rhabdoiden nicht nur bedeutungsvoll für die Systematik, sondern sie sind auch am besten geeignet, die phylogenetischen Beziehungen innerhalb der Actiniaria und Anthozoa aufzuzeigen. Soweit den Mitteilungen Carlgrens (1940) zu entnehmen ist, fehlen die Kategorien A und C den Unterordnungen Protantheae und Endocoelantheae. Innerhalb der Stämme der dritten Unterordnung der Actiniaria, den Nynantheae, weisen nach dem bisherigen Stand unserer Kenntnisse über das Cnidom der verschiedenen Familien und Gattungen die Boloceroidaria (einschließlich der Aliciidae) die Kategorien $B$ und $C$ und die Athenaria und Thenaria die Kategorien $A$ und $B$ auf. Ich stimme mit HAND (1966) darin überein, daß die Ansicht, aus den Athenarien seien die Thenarien hervorgegangen, nicht den wirklichen phylogenetischen Verhältnissen gerecht wird. Ich kann aber an- 
dererseits nicht die Auffassung Hands teilen, daß die Athenarien den Thenarien entstammten. Beide Stämme setzen sich aus so verschiedenartigen Familien zusammen, daß ihre systematische Bedeutung fragwürdig ist. Abgesehen von dem bisherigen Hauptunterscheidungsmerkmal, der $\mathrm{Ab}-\mathrm{bzw}$. Anwesenheit einer basilaren Muskulatur, sind zum Beispiel in beiden Stämmen Formen vertreten, die entweder über Akontien und einen mesogloealen Sphinkter oder nur über einen entodermalen Sphinkter verfügen. So haben die Andresiidae (Athenaria) einen entodermalen Sphinkter aber auch ausschließlich p-Rhabdoiden der Kategorie A wie der überwiegende Teil der Actiniidae (vgl. Stephenson 1921).

Aus den Mitteilungen Carlgrens (1940, 1949) geht hervor, daß die Andwakiidae (Athenaria) ebenso Akontien, einen mesogloealen Sphinkter und lediglich p-Rhabdoiden der Kategorie B aufweisen wie zum Beispiel die Aiptasiidae innerhalb der Thenaria (Tab. 3). Leider ist das Cnidom vieler Aktinien insbesondere der Athenaria noch zu wenig bekannt, um endgültige Aussagen über die Verwandtschaftsbeziehungen der verschiedenen Familien beider Stämme zu machen.

Der geringgradige oder fehlende Unterschied im Kaliber zwischen entladenem Schaft und Faden läßst die b-Rhabdoiden oder Aktinien näher mit den Haplonemen als mit den p-Rhabdoiden verwandt erscheinen, deren Faden außerdem unbewaffnet ist. Ein Vergleich mit den Nesselkapseln anderer Anthozoen schließt diese Vermutung allerdings aus. Die b-Rhabdoiden der Ceriantharien verfügen über einen ähnlich erweiterten Schaft wie die p-Rhabdoiden der Aktinien (CARLGREN 1940). Die p-Rhabdoiden der Corallimorphen und Madreporarien haben im Gegensatz zu den p-Rhabdoiden der Aktinien einen deutlich bewaffneten Faden (CARLgren 1940, RoBson 1953). HaND (1966) leitet aber die Actiniaria von den Madreporaria ab, da insbesondere die Actiniidae gleiche Nesselkapseln und gleiche Sphinkteren aufweisen sollen wie die Korallen. An Corynactis viridis und verschiedenen Madreporarien ${ }^{2}$ aus dem Mittelmeer konnte ich eine gewisse Ahnlichkeit der p-Rhabdoiden mit der Kategorie A der Aktinien feststellen. Bei den besonders großen p-Rhabdoiden der Madreporarien und Corallimorphen treten nach der Entladung keine Klappen hervor und die Kapsel ist ebenfalls ziemlich dünnwandig. Die Dornenwindungen sind allerdings wesentlich dichter (vgl. Robson 1953, Fig. 2E). Besser stimmt die Kategorie A der Aktinien mit den p-Rhabdoiden der Zoantharien überein, wie ich an Parazoanthus axinellae und Gerardia savaglia feststellen konnte; die senkrecht zur Schaftoberfläche stehenden Dornen sind ebenfalls in deutlich abgesetzten Spiralen angeordnet (vgl. WeILI 1934, Fig. 431). Außerdem konnte ich an der dünnwandigen Kapsel im Phasenkontrastmikroskop keine Klappen und an dem Faden keine Bewaffnung erkennen (vgl. WeILL 1934, p. 621). Dieser Befund muß allerdings noch elektronenmikroskopisch nachgeprüft werden. In Ubereinstimmung mit den p-Rhabdoiden der Kategorie A der Aktinien sind außerdem die p-Rhabdoiden der Zoantharien im Gegensatz zu denjenigen der Madreporarien nur auf die Mesenterialflamente beschränkt. Eine vergleichende Betrachtung ergibt, daß die Dornen der p-Rhabdoiden der Corallimorphen (RoBsoN 1953) und der Aktinien große Ahnlichkeit besitzen. Gleiches wird auch bei elektronenmikroskopischer Betrachtung für die p-Rhabdoiden der Madreporarien, Antipatharien

2 Mademoiselle F. Lafargue, Banyuls, danke ich für die liebenswürdige Oberlassung von acht Arten. 
und Zoantharien zutreffen. Die Form der Haplonemendornen der Corallimorphen weicht dagegen deutlich ab (SKAER \& PICKeN 1965). Es bestehen daher keine Beziehungen zwischen den Haplonemen der Corallimorphen und der Kategorie C der p-Rhabdoiden der Aktinien, die Cutress (1955) in der Kategorie der makrobasischen p-Mastigophoren vereinte.

Die Untersuchungen von WesTFALl (1966) haben ergeben, daß sich die Nesselkapseln der Anthozoen grundsätzlich durch das Auftreten von drei Klappen und eines Flagellum anstelle eines Operculum bzw. Cnidocils von den Nesselkapseln der Hydround Scyphozoen unterscheiden. Bei einigen dünnwandigen $\mathrm{p}$-Rhabdoiden konnte ich allerdings weder Klappen noch Operculum feststellen. Der Befund Westfalxs wirft jedoch die Frage auf, ob sich die Nesselkapseln der verschiedenen Cnidarierklassen überhaupt in einem System vereinen lassen. In einem allgemein gültigen System für alle Cnidarier können daher nur sehr allgemeine Kriterien bei den Diagnosen der einzelnen Kategorien berücksichtigt werden. Daraus folgt, daß die Anwendung eines derartigen Systems auf die höheren taxonomischen Einheiten beschränkt bleibt. Für die niedrigeren systematischen Niveaus mïssen spezielle Unterteilungen eingeführt werden (vg1. WERnER 1965). Die unterschiedliche Bewaffnung und die damit verbundenen Querschnittsunterschiede am $n$ ich $t$ entladenen Schlauch können zur Charakterisierung der Nesselkapseln aller Cnidarierklassen herangezogen werden, da WESTFALL (1966b) z. B. auch bei den Hydrozoen (Obelia longissima) an Werlus Basitrichen einen bewaffneten Faden beobachtete. Daher fordern die vorliegenden Befunde folgende Abänderung des WeIllschen Systems (vgl. WeIll 1934, Werner 1965):

(I) $\mathrm{H}$ a p lo n e men : Schlauch einheitlich, ohne abgesetzten proximalen Schaft oder abgesetzte Bewaffnung.

(1) Isorhizen: Schlauch weitgehend isodiametrisch;

(2) Anisorhizen: Schlauch an der Basis deutlich erweitert.

(II) Heteronemen: Schlauch mit deutlich abgesetzter Bewaffnung, daher unterteilbar in Schaft und Faden.

(1) b-Rhabdoiden: Schaft in der Kapsel ohne distale trichterförmige Offnung, Faden bewaffnet;

(2) p-Rhabdoiden: Schaft in der Kapsel mit distaler trichterförmiger Offnung;

(a) hoplotelisch: Faden bewaffnet;

(b) anoplotelisch: Faden unbewaffnet.

Die Diagnosen der verschiedenen Kategorien sind mit Ausnahme derjenigen der b- und p-Rhabdoiden so gestellt, daß eine Bestimmung der meisten Nesselkapseln sowohl in entladenem wie in nicht entladenem Zustand möglich ist. CarLgrens (1940) Behauptung, die p-Rhabdoiden würden sich grundsätzlich durch einen deutlichen Konus am distalen Ende des entladenen Schaftes von den b-Rhabdoiden unterscheiden, trifft nicht für die Kategorie A der Aktinien zu (Abb. 8). Daher sind die b- und $\mathrm{p}$-Rhabdoiden nur in unentladenem Zustand zu diagnostizieren, sieht man von feineren Unterschieden, wie der verschiedenartigen Form der Dornen ab. Den Abbildungen WemLs (1934, p. 470) ist zu entnehmen, daß es auch bei den Hydrozoen Rhabdoiden mit und ohne trichterförmige Schaftöffnung gibt. So stellt seine Abbildung 300 eine typische p-Rhabdoide dar, während die Nesselkapseln auf den Abbildungen 294 bis 296 und 306 eher b-Rhabdoiden gleichen, die teilweise (Fig. 296, 306) auch einen er- 
weiterten entladenen Schaft zeigen. Abbildung 293 zeigt sogar eine „Basitriche“, die in entladenem Zustand keinen Querschnittsunterschied zwischen Schaft und Faden aufweist, in nicht entladenem Zustand aber über eine trichterförmige Offnung am distalen Schaftende verfügt. Derartige $\mathrm{p}$-Rhabdoiden sind allerdings nicht wieder beobachtet worden. Solange Werl s. Befunde jedoch nicht widerlegt sind, wie z. B. bei den b-Rhabdoiden von Metridium (CARLgREN 1940), muß angenommen werden, daß auch die Hydrozoen über b- und $\mathrm{p}$-Rhabdoiden verfügen.

Sollten sich Werlus Befunde dagegen nicht bestätigen lassen, so beginnt die Spezialisierung des vorliegenden Systems bereits bei der Unterteilung der Rhabdoiden, die ohnehin den Scyphozoen fehlen (WERnER 1965, Tab. 2).

Obwohl der Vorschlag eine wesentliche Vereinfachung des WeILLschen Systems darstellt, sinkt damit nicht die Bedeutung für die Systematik. Bei den Hexakorallen lassen sich nach dem bisherigen Stand unserer Kenntnisse Ceriantharien (keine p-Rhabdoiden), Madreporarien (hoplotelische $\mathrm{p}$-Rhabdoiden) und Actiniarien (anoplotelische p-Rhabdoiden) eindeutig unterscheiden. Der unterschiedliche Bau einiger Nesselkapseltypen läßt eine weitere spezielle Unterteilung $z u$, die für die unteren taxonomischen Einheiten von großer diagnostischer Bedeutung sein können, wie am Beispiel der p-Rhabdoiden der Actiniaria gezeigt wurde.

\section{ZUSAMMENFASSUNG}

1. Die Ergebnisse von phasen- und elektronenoptischen Untersuchungen an Nesselkapseln von 36 Aktinienarten aus Nordsee, Mittelmeer und Rotem Meer erfordern eine Abwandlung des Systems yon WerLL (1934).

2. Nematocysten und Spirocysten weichen in ihrem Bau voneinander ab. Der unbewaffnete Faden der Spirocysten wird im Gegensatz zu den Nematocysten unmittelbar nach der Entladung von einem einzigen zusammenhängenden Sekretfaden (Quellfaden) in Linkswindungen umgeben.

3. Bei den Nematocysten bestehen enge Beziehungen zwischen der Form des $\mathrm{n}$ i $\mathrm{ch}$ entladenen Schlauches und der Bewaffnung. In nicht entladenem Zustand unterscheiden sich die Heteronemen von den Haplonemen durch einen abgesetzten proximalen Schaft (Achsenkörper), in entladenem Zustand durch eine abgesetzte Bewaffnung.

4. Die von WeILl (1934) gegebene Einteilung in isorhize und anisorhize Haplonemen ist aus taxonomischen Gründen sinnvoll. Eine weitere Unterteilung ist dagegen nicht möglich. Haplonemen sind bei den Aktinien weiter verbreitet als bisher bekannt. Im Scapus verschiedener Aktinienarten konnten sie als konstanter Bestandteil des Cnidoms nachgewiesen werden. Bei einigen Hormathiiden zeichnen sich einzelne Individuen durch massenhaftes Auftreten von Haplonemen in allen Tentakeln aus. Die Tentakeln weisen hingegen keine morphologischen Abweichungen auf, wie die Fangtentakeln anderer Acontiaria.

5. Die bisherige Einteilung der rhabdoiden Heteronemen in mikrobasische und makrobasische Mastigophoren und Amastigophoren ist nicht mit den vorliegenden Befunden zu vereinbaren. Weder die Schaflänge noch die Ausstullpung des Fadens 
eignen sich zur Einteilung der Rhabdoiden, sondern stellen Merkmale von untergeordneter taxonomischer Bedeutung dar.

6. Nach CARLgren (1940) werden b- und p-Rhabdoiden unterschieden. Bei den p-Rhabdoiden stülpt sich der Faden im Gegensatz zu den b-Rhabdoiden am Ende der Reifungsphase durch eine ringförmige Wandverdickung in die trichterförmige distale Schaftöffnung ein. Beide Kategorien haben verschiedenartige Dornen.

7. In Übereinstimmung mit den Ceriantharien sind sämtliche b-Rhabdoiden der Aktinien mit einem bewaffneten Faden versehen (hoplotel). Im Gegensatz zu den Madreporarien und Corallimorphen ist dagegen der Faden sämtlicher p-Rhabdoiden bei den Aktinien unbewaffnet.

8. Auf Grund der verschiedenartigen Struktur und Bewaffnung kann am Schaft einiger Rhabdoiden ein proximales Faltstück und ein distales Hauptstück unterschieden werden. Der Schaft der übrigen Rhabdoiden gleicht dem Hauptstück.

9. Die Entladung der Nesselkapseln wird durch Erhöhung des Kapselinnendruckes ausgelöst. Wird die Ausstülpung des Schaftes behindert, so werden die Dornen im Zusammenhang aus ihrer Verankerung gelöst und als pfeilartiges Gebilde ausgeworfen. Der Vorgang ist bei b- wie bei $\mathrm{p}$-Rhabdoiden zu beobachten, die in nicht entladenem Zustand über einen geradlinigen Schaft mit elastischem Faltstück verfügen.

10. Die b-Rhabdoiden stellen bei den Aktinien eine ziemlich einheitliche Kategorie dar. Aus den Akontien der Aiptasiiden, den Mesenterialfilamenten einiger Actiniiden und dem Scapus yon Paranthus werden neue Varianten beschrieben.

11. Die p-Rhabdoiden der Aktinien bestehen aus drei Kategorien, die für Taxonomie und Phylogenie der Actiniaria bedeutungsvoll sind, wie an einigen Beispielen gezeigt wird. Die Hauptunterschiede der einzelnen Kategorien bestehen im Phasenkontrast des Kapselinhaltes, in der Wandstärke und im Verschluß der Kapsel sowie in der Struktur und der Bewaffnung des Schaftes.

Danksagungen. Herrn Prof. Dr. Dr. h. c. W. E. ANkel danke ich für die Durchsicht des Manuskriptes und kritische Diskussion. Mein Dank gebührt weiterhin Herrn Prof. Dr. A. Cecro und Herrn Dr. D. Zissler für die Anleitung zur Elektronenmikroskopie. Das Material wurde mit freundlicher Unterstützung der Meeresbiologischen Stationen von Banyuls, Dubrovnik, Helgoland und Neapel sowie der Zoologischen Institute der hebräischen Universität von Jerusalem und der Universität von Palermo besorgt. Die Arbeit wurde mit dankenswerter Förderung durch die VW-Stiftung und den DAAD ausgeführt.

\section{ZITIERTE LITERATUR}

Andres, A., 1884. Attinie. Fauna Flora Golf. Neapel 9, 1-459.

Bonnenfant, J., 1960. Recherches sur l'évolution larvaire et la variation physiologique du cnidome chez Actinia equina. Bull. Soc, zool. Fr, 85, 157-165.

BRETsChNEIDER, L. H., 1949. A simple tednique for electron-microscopy of cell and tissue sections. Proc. K. ned. Akad. Wet. 52, 654-666.

Carlgren, O., 1925. On Boloceroides, Bunodeopsis and their supposed allied genera. Ark. Zool. 17 A (1), 1-20. 
CaRlgren, O., 1929. Uber eine Actiniariengattung mit besonderen Fangtentakeln. Zool. Anz. 81, 109-113.

- 1940. A contribution to the knowledge of the structure and distribution of the cnidae in the Anthozoa. Lunds Univ. Arsskr. N. F. (Avd. 2) 36 (3), 1-62.

- 1945. Further contribution to the knowledge of the cnidom in the Anthozoa, especially in the Actiniaria. Lunds Univ. Ärsker. N. F. (Avd. 2) 41 (9), 1-24.

- 1949. A survey of the Ptychodactiaria, Corallimorpharia and Actiniaria. K. suenska Vetensk-Akad. Handl. 1 (1), 1-121.

Cutress, C. E., 1955. An interpretation of the structure and distribution of cnidae in Anthozoa. Syst. Zool. 4, 120-137.

GünzL, H., 1968. Über die Reifung der Nesselkapseln bei Dipurena reesi Vannuccr (Hydrozoa). Z. Zellforsch. mikrosk. Anat. 89, 509-518.

HAnd, C., 1955. The sea anemones of central California. III. The acontiarian anemones. Wasmann J. Biol. 13, 189-251.

- 1961. Present state of nematocyst research: Types, structure and function. In: Biology of Hydra and some other coelenterates. Ed. by H. M. Lenhoff \& W. F. Loomis. Univ. of Miami Press, Coral Gables, Fla, 187-202.

- 1961a. Two new acontiate New Zealand sea anemones. Trans. R. Soc. NZ (Zool.) 1 (4), $75-89$.

- 1966. On the evolution of the actiniaria. In: The Cnidaria and their evolution. Ed. by W. J. Rees. Acad. Press, London, 135-146. (Symp. zool. Soc. Lond. 16.)

Iwanzoff, N., 1896. Uber den Bau, die Wirkungsweise und die Entwidklung der Nesselkapseln der Coelenteraten. Byull. mosk. Obshch. Ispyt. Prir. (Bull. Soc. Nat., Moscou) 1, 95-161.

Mergner, H., 1964. Unsere Kenntnisse über Bau und Funktion der Nesselzellen bei Hydroiden. Natur. Mus., Frank. 94, 15-32.

MrLNE EDWARDS, H., 1857. Histoire naturelle des coralliaires ou polypes proprement dits. Paris, 1, 225-298.

Picken, L. E. R., 1953. A note on the nematocysts of Corynactis viridis. Q. J. microsc. Sci. 94, 203-227.

- \& Skatr, R. J., 1966. A review of researches on nematocysts. In: The Cnidaria and their evolution. Ed. by W. J. Rees. Acad. Press, London, 19-50. (Symp. zool. Soc. Lond. 16.)

Rosson, E. A., 1953. Nematocysts of Corynactis: The activity of the filament during discharge. Q. J. microsc. Sci. 94, 229-235.

Russer, F. S., 1953. The medusae of the British isles. Univ. Press, Cambridge, 530 pp.

Schmid, H, 1967. A note on the sea-anemones Bunodactis rubripunctata GRuBE and Aulactinia crassa Andres. Pubbl. Staz. zool. Napoli 35, 250-251.

- 1967a. A note on the sea-anemones Bunodactis verrucosa Pennant. Pubbl. Staz. zool. Napoli 35, 252-253.

Schnerder, K. C., 1900. Mittheilungen über Siphonophoren. V. Nesselzellen. Arb. zool. Inst. Univ. Wien 12, 1-110.

Skaer, R. J. \& PICKen, L. E. R., 1965. The structure of the nematocyst thread and the geometry of discharge in Corynactis viridis Allman. Pbil. Trans, R. Soc. (B) 250 (764), $131-164$.

Stephenson, T. A., 1921. On the classification of Actiniaria. II. Consideration of the whole group and its relationships, with special reference to forms not treated in part $I . Q . J$. microsc. Sci. 65, 493-576.

- 1929. On the nematocysts of sea anemones. J. mar. biol. Ass. U. K. 16, 173-201.

- 1935. The British sea anemones. Adlard \& Son, London, 2, 1-426. (Monogr. Ray Soc. 121.)

Wenl, R., 1934. Contribution a l'étude des Cnidaires et leur nématocystes. I. Recherches sur les nématocystes. II. Valeur taxonomique du cnidom. Trav. Stn zool. Wimereux 10/11, $1-701$.

WeRnER, B., 1965. Die Nesselkapseln der Cnidaria, mit besonderer Berücksichtigung der Hydroida. I. Klassifikation und Bedeutung für die Systematik und Evolution. Helgoländer wiss. Meeresunters. 12, 1-39. 
Westfall, J. A. \& Hand, C., 1962. Fine structure of nematocysts in a sea anemone. Int. Conf. Electron Microsc. 5 (Philadelphia), M-13.

Westpali, J. A., 1964. Fine structure and development of nematocycsts in the tentacle of Metridium. Am. Zool. 4, 435.

- 1965. Nematocysts of the sea anemone Metridium. Am. Zool. 5, 377-393.

- 1966. The differentiation of nematocysts and associated structures in the Cnidaria. Z. Zellforsch. mikrosk. Anat. 75, 381-403.

- 1966a. Fine structure and evolution of nematocysts. Int. Conf. Electron Microsc. 6 (Tokyo), 235-236.

- 1966b. Electron microscopy of the basitrich and its associated structures in Obelia. Am. Zool. 6, 554.

WiLL, L., 1909. Die Klebkapseln der Aktinien und der Mechanismus ihrer Entladung. Sber. Abb. naturf. Ges. Rostock 1, 65-103.

- 1914. Kolloidale Substanz als Energiequelle für die mikroskopischen Schußwaffen der Coelenteraten. Abh. preuß. Akad. Wiss. (Phys.-math. Kl.) 1, 1-28.

Anschrift des Autors: Dr. Hajo Schmidr

I. Zoologisches Institut

der Universität Gießen

63 Gießen

Stephanstr. 25 